\title{
Computational Fluid Dynamics-based Transonic Flutter Suppression with Control Delay
}

\author{
Qiang Zhou ${ }^{a, b}$, Dong-feng $\mathrm{Li}^{a, b}$, Andrea Da Ronch ${ }^{c}$, Gang Chen ${ }^{a, b, *}$, Yue-ming Li $\mathrm{Li}^{a, b}$ \\ a. State Key Laboratory for Strength and Vibration of Mechanical Structures, School of \\ Aerospace, Xi'an Jiaotong University, Xi'an, 710049, China \\ b. Shannxi Key Laboratory for Environment and Control of Flight Vehicle, School of \\ Aerospace, Xi'an Jiaotong University, Xi'an, 710049, China \\ c. Faculty of Engineering and the Environment, University of Southampton, Southampton, \\ SO17 IBJ, U.K.
}

\begin{abstract}
This work investigates the effects of control input time delay on closed-loop transonic computational aeroelastic analysis. Control input time delays are becoming critical as the demand for high frequency control actions is increasing. The flow in transonic conditions exhibits strong nonlinearities which require accurate physical modelling techniques, in turn resulting in large dimensional systems that are computationally costly to solve. A unified framework is demonstrated for the robust and efficient generation of reduced order models. Once generated, the reduced order model is employed for the flutter boundary search, and excellent agreement with the large order coupled model is demonstrated. The aero-servo-elastic reduced order model is then exploited to design a feedback control law, which is implemented in the fully coupled computational fluid/structural dynamics solver. As expected, the controller effectiveness is found to degrade for increasing time delay, up to a critical value where the controller fails to suppress flutter. It is shown that a controller for a time-delay system may be designed using the same aero-servo-elastic reduced order model, incurring in no extra costs or complications. The new controller is found to achieve excellent flutter suppression characteristics. The aero-servo-elastic reduced order model may also be used to identify, for a given feedback controller, the critical value of control input time delay at which the closed-loop aero-servo-elastic system loses its stability. The test cases are for a two-dimensional pitch-plunge aerofoil section and the AGARD 445.6 wing modified with a trailing-edge control surface.
\end{abstract}

Keywords: Transonic aeroelasticity, time delay, flutter suppression, CFD, POD, ROM, LQR, AGARD 445.6

\footnotetext{
* Corresponding author.

E-mail address: aachengang@ mail.xjtu.edu.cn (Prof Gang Chen), A.Da-Ronch@ soton.ac.uk (Dr Andrea Da Ronch),
} 


\section{Introduction}

Flutter is a dynamic instability phenomenon caused by the interaction of aerodynamic, elastic and inertial forces. At flutter, a catastrophic failure of the airframe will normally occur. To control or suppress flutter ensuring the structural integrity of the aircraft structural components, several active and passive control strategies have been investigated (Da Ronch et al., 2013b; Marzocca et al., 2012; Papatheou et al., 2013). In this work, in particular, the interest is on active control strategies that are relevant to industrial aircraft design, extending the operational flight envelope and ensuring safety. Among active control techniques, a large body of work using feedback strategies has been reported, see for example (Da Ronch et al., 2013b; Tantaroudas et al., 2015). Adaptive and nonlinear feedback control has been investigated, for example, in (Tantaroudas et al., 2014) where a numerical study for gust loads alleviation of various aeroelastic models was presented, and Refs. (Da Ronch et al., 2014; Fichera et al., 2014; Papatheou et al., 2013)demonstrated the suppression of limit cycle oscillations (LCOs) for a nonlinear aeroelastic wind tunnel model. On the other hand, feedforward control strategies have the advantage that there is no time delay between the disturbance measurement and the control action. Whereas a number of numerical investigations (Wang et al., 2014; 2015) showed promising results compared to feedback control, the measurement of the disturbance is challenging in an experimental setting, particularly to detect atmospheric turbulence in flight.

Several classic and modern control theories have been applied to design active feedback control laws, such as linear quadratic regulator (LQR) and optimal controllers. However, these control strategies may be ineffective if the aeroelastic system has a control delay. Time delays in control loops are inevitable when digital controllers and hydraulic actuators are modelled (Liu and Hu, 2010), and may arise from measuring and filtering the system states, computing the control outputs, and transmitting control outputs to actuators. Time delays become particularly critical when the control effort demands large control forces or high frequencies. Time delay introduces a phase shift, which deteriorates the controller performance and may drive the system to instability. In most previous studies in aero-servo-elasticity, time delays in control feedback loops have been neglected. In some cases, a suitable control delay was found to improve the system stability (Zhao, 2009; Zhao and Xu, 2007). In Ref. (Olgac and Holm-Hansen, 1994), a delayed feedback control was investigated. The 
technique offers a number of attractive advantages in eliminating oscillations of the primary system, such as real time tunability, wide range of frequency, and simplicity of the control. For a suitable delay in the control feedback loop system, the delay feedback control may expand the system's stability region (Zhao, 2009). References (Zhao, 2009, 2011) presented a systematic study on aeroelastic stability of a twodimensional (2D) aerofoil in incompressible flow with single or multiple time delays in the feedback control loops. It was found that a small time delay in the feedback control can stabilize the aeroelastic system, which is initially unstable under delayfree control.

Stability analysis and control of time delayed systems have been widely studied in recent years (Chen and Xu, 2013; Dai et al., 2014c, 2015; Hu et al., 1998a, b). In the field of aero-servo-elasticity, for example, (Yuan et al., 2004) and (Xu et al., 2014) investigated the effects of the time-delayed feedback control on the flutter instability boundary of a 2D supersonic lifting surface. Reference (Librescu et al., 2005) presented the effects of time delay on the stability of a $2 \mathrm{D}$ aeroelastic system in incompressible flow and designed a delayed feedback controller to stabilize the system. Previous studies have mainly focused on a 2D aeroelastic system, with few cases looking at three-dimensional (3D) aeroelastic models. For example, (Huang et al., 2012; Huang et al., 2015b) presented numerical and experimental studies on the active flutter suppression (AFS) of a 3D wing model involving a control delay. A high-dimensional multiple-actuated-wing was established in their work as a test case to validate the control method.

Generally, aero-servo-elastic studies have used low-fidelity linear aerodynamic models, including lifting surface theory (Yuan et al., 2004), piston theory (Xu et al., 2014), quasi-steady aerodynamics (Zhao, 2009, 2011), and the doublet-lattice method (Huang et al., 2012; Huang et al., 2015b). The assumption of linear aerodynamics is adequate to treat subsonic and supersonic flow regimes (Xie et al., 2014a). In transonic conditions, which are relevant for passengers' transport aircraft jets, the flow is dominated by nonlinear effects and exhibits complex interactions between shock waves and boundary layer (Alder, 2015; Dai et al., 2014a; Dai et al., 2014b). Take-off and landing conditions are equally critical phases of flight, in particular when in presence of cross-winds. Computational fluid dynamics (CFD) solvers are needed for realistic predictions in transonic conditions. From the available literature, 
it is apparent that the effects of time delay and delayed feedback control designed for transonic aeroelastic models have been rarely studied.

With the previous paragraphs as background, this paper aims at investigating time delay effects in transonic aeroelastic models, and developing control strategies for the transonic flutter suppression. High-fidelity aeroelastic modelling, based on coupling a research CFD solver with a computational structural dynamics (CSD) solver, is used for accurate time marching. Two considerations are worth noting about the application of coupled CFD/CSD methods for AFS problems: first, the computational cost of coupled simulations for 3D configurations is today unrealistic for practical applications despite the availability of high performance computing (HPC) facilities ${ }^{1}$; second, a low-dimensional state space model is needed for the control design synthesis. To find a compromise between these two contrasting requirements, model reduction techniques aim at balancing high fidelity and low cost/dimensionality. Various techniques exist, but these are generally limited to linear or weakly-nonlinear systems (Ghoreyshi et al., 2013; Lucia et al., 2004). Among nonlinear ROMs, the harmonic balance (Da Ronch et al., 2013a) and the nonlinear model projection (Da Ronch et al., 2012; Da Ronch et al., 2013c; Timme et al., 2013) have been applied to a variety of test cases and models. The latter method, in particular, is well suited to control synthesis design for gust loads alleviation. Alternative methods based on proper orthogonal decomposition (POD) (Xie et al., 2014b) have also been used to generate CFD-based ROMs. References (Chen et al., 2012b) and (Chen et al., 2014), respectively, performed AFS and control design for gust loads alleviation using a POD ROM, and a balanced truncation (BT) method was applied to further reduce the dimension of the time-domain POD ROM.

In this work, the impact of control input time delay on the transonic stability of two aero-servo-elastic test cases is investigated, and feedback control is implemented to enhance the stability of the system. Reduced order models are generated from the coupled CFD/CSD models for control design synthesis. The paper continues in Section 2 with a description of the CFD/CSD aero-servo-elastic model and the corresponding ROM based on the BT method combined with POD. Section 3

\footnotetext{
1 The University of Southampton hosts two HPC facilities, Iridis4 and Lyceum. Iridis4, in particular, is ranked the most powerful academic supercomputer in the UK with 12,320 Intel® Xeon ${ }^{\circledR}$ E5-2670 processor cores, a petabyte of disc space, and 50 terabytes of memory.
} 
summarises the control design approach, whereby the controller is designed on the aero-servo-elastic ROM with time delay and then applied for verification on the largedimensional CFD/CSD aero-servo-elastic model. Section 4 overviews the test cases for a 2D pitch-plunge aerofoil section and the AGARD 445.6 wing, and provides a validation of both CFD/CSD and ROM approaches. Then, Section 5 presents the closed-loop aeroelastic responses computed above the flutter point, and the effect of time delay on the performance of the feedback controller to suppress the instabilities. Finally, conclusions are given in Section 6.

\section{Aero-servo-elastic State Space Model}

The starting point is the coupled CFD/CSD model, herein referred to as the full order model (FOM). The FOM has the capability to provide physical insights on transonic aero-servo-elasticity at the cost of solving a large dimensional problem. The coupled problem is formulated as

$$
\begin{gathered}
\frac{d(\mathbf{A} \cdot \mathbf{w})}{d t}+\mathbf{F}(\mathbf{w})=\mathbf{0}_{N_{f}} \\
\mathbf{M} \frac{d^{2} \mathbf{X}}{d^{2} \tau}+\mathbf{G} \frac{d \mathbf{X}}{d \tau}+\mathbf{K X}=\mathbf{f}
\end{gathered}
$$

The first set of equations corresponds to a finite volume discretization of the NavierStokes equations adopting an arbitrary Lagrangian-Eulerian (ALE) formulation. The second set of equations is for a (linear) finite element (FE) discretization of the structural dynamic equations. The vector of conservative flow variables is denoted by $\mathbf{w}$, the volume of the fluid cell is denoted by $\mathbf{A}$, and $\mathbf{F}$ indicates in this work the inviscid flux resulting from the spatial discretisation. For the structural model, $\mathbf{M}, \mathbf{G}$, and $\mathbf{K}$ indicate, respectively, the mass, damping, and stiffness matrices. Finally, $\mathbf{f}$ is the vector of aerodynamic forces computed from Eq.(1). Characteristic phenomena in fluid and structural dynamics occur over different time scales, and two different temporal discretisations have been used above: $t$ for the fluid, and $\tau$ for the structural part. The fluid and structural models are highly coupled through: $a$ ) the dependency of the conservative flow variables, $\mathbf{w}$, on structural deformations, $\mathbf{X}$, and corresponding velocities; and $b$ ) the dependency of the structural deformations on the applied aerodynamic loads, $\mathbf{f}$. 


\subsection{Flow and Structural Solvers}

In this work, the Euler equations are solved on a structured mesh. Neglecting the viscous effects is considered a reasonable assumption for the high Reynolds number of the transonic flow conditions tested in this paper. The CFD solver uses a cellcentred finite volume formulation and the spatial discretization is based on the second-order Van Leer scheme (Van Leer, 1979). For time marching, the lower-upper symmetric Gauss-Seidel (LU-SGS) method is used. An interpolation method based on the interpolation using surface splines (IPS) (Harder and Desmarais, 1972) allows transferring applied forces from the aerodynamic domain to the structural domain. The aerodynamic mesh deformation is obtained by using radial basis functions (RBF) combined with the transfinite interpolation (TFI) method (Tsai et al., 2001; Yingtao et al., 2013). Flutter analysis is studied as a linear instability problem around a nonlinear equilibrium. For the treatment of the control surface in the CFD model, gaps between the control surface and the main wing are neglected. The motion of the control surface is modelled by an appropriate structural mode-shape. The corresponding (structural) modal displacement is mapped to the aerodynamic surface mesh to simulate the rotation of the control surface using the IPS method.

The structural problem is solved using a FE approach. Modal superposition is used to calculate the structural dynamic response. Once the first $n$-th mode-shapes are calculated, the structural deformations, $\mathbf{X}$, may be expressed as $\mathbf{X}=\mathbf{\Phi u}$, where $\mathbf{u}$ is the modal displacement vector, $\mathbf{u} \in \mathbf{R}^{n \times 1}$. Equation (2) in modal coordinates becomes

$$
\overline{\mathbf{M}} \cdot \frac{d^{2} \mathbf{u}}{d^{2} \tau}+\overline{\mathbf{G}} \cdot \frac{d \mathbf{u}}{d \tau}+\overline{\mathbf{K}} \cdot \mathbf{u}=Q=q \cdot\left(\mathbf{f}^{e x t}-\mathbf{f}_{0}^{e x t}\right)
$$

where $\overline{\mathbf{M}}=\boldsymbol{\Phi} \mathbf{M}, \overline{\mathbf{G}}=\boldsymbol{\Phi} \mathbf{G} \boldsymbol{\Phi}, \overline{\mathbf{K}}=\boldsymbol{\Phi} \mathbf{\Phi} \boldsymbol{\Phi}$, and $q$ is dynamic pressure $q=1 / 2 \rho \mathrm{V}_{f}^{2}$. This set of equations is easily recast in state-space form

$$
\left\{\begin{array}{l}
\dot{\mathbf{x}}_{s}(\tau)=\mathbf{A}_{s} \cdot \mathbf{x}_{s}(\tau)+q \cdot \mathbf{B}_{s} \cdot \mathbf{f}^{e x t}(\tau) \\
\mathbf{u}=\mathbf{C}_{\mathrm{s}} \cdot \mathbf{x}_{s}(\tau)
\end{array}\right.
$$

where, for convenience, the over-dot is used to indicate the time derivative, and

$$
\mathbf{A}_{s}=\left[\begin{array}{cc}
\mathbf{0} & \mathbf{I} \\
-\overline{\mathbf{M}}^{-1} \overline{\mathbf{K}} & -\overline{\mathbf{M}}^{-1} \overline{\mathbf{G}}
\end{array}\right], \mathbf{B}_{s}=\left[\begin{array}{c}
\mathbf{0} \\
\overline{\mathbf{M}}^{-1}
\end{array}\right], \mathbf{C}_{s}=\mathbf{I}_{n}, \mathbf{x}_{s}=[\mathbf{u}, \dot{\mathbf{u}}]^{\mathrm{T}}
$$

The vector of modal coordinates is indicated by $\mathbf{x}_{\mathrm{s}}$, and $\tau$ indicates the physical time governing the structural dynamic equations. 


\subsection{CFD/CSD coupling procedure}

A fully implicit partitioned approach is used for time-domain coupled CFD/CSD calculations. The solution sequencing between the fluid and the structural models is achieved within the pseudo-time stepping iterations. The models are sequenced in pseudo-time towards perfect sequencing in real time. The proposed approach improves the numerical stability compared to a staggered procedure, in which separate analysis codes are run in a strictly sequential manner. First, a converged steady CFD solution is obtained around the un-deformed fluid mesh. This base solution is the starting point of the coupled CFD/CSD simulation. An initial condition on the velocity of the first structural mode-shape is imposed, and the structural displacements are then computed by the CSD solver. The displacements on the structural domain are mapped to the CFD surface mesh through the IPS method. For the volume mesh deformation, the RBF method combined with TFI is used. The aerodynamic loads are recomputed on the deformed CFD mesh. The aerodynamic loads from the fluid domain are transferred to the structural domain using the IPS method. Once the structural deformations are recomputed by the CSD solver, the iterative process is repeated until convergence. The coupling procedure is illustrated in Fig. 1. Two processes are indicated: $a$ ) open-loop, whereby the control surface is not activated (rotated) during the analysis; and $b$ ) closed-loop, whereby the control surface rotation is updated at each time step by a predefined control law.

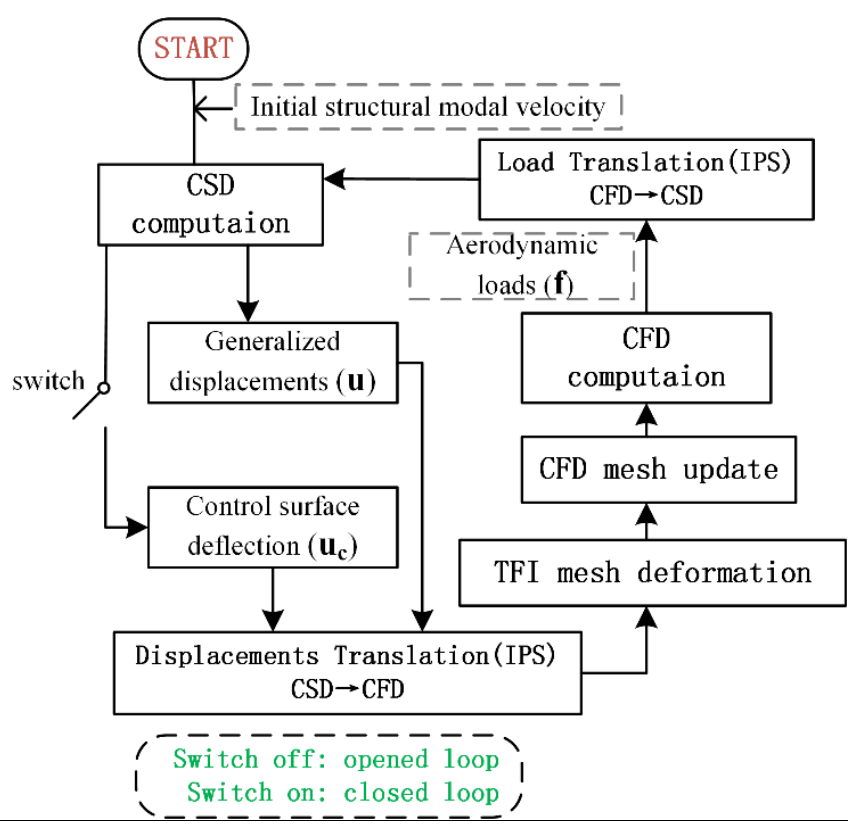

Fig. 1 CFD/CSD coupling procedure for open- and closed-loop aeroelastic analysis 


\subsection{Proper orthogonal decomposition (POD) method}

The POD method provides a basis to accurately represent a given data set. The basis can be deemed a low-order model of the original FOM. For one series of data, $\left\{\mathbf{x}_{k}\right\}, k=1 \ldots m$, which is called snapshot in the POD method, $\mathbf{x}_{\mathrm{k}}$ is in the $n$ dimensional space, and $m$ is the number of snapshots. The POD method searches an $m$-dimensional proper orthogonal subspace, $\boldsymbol{\Psi} \in \mathbf{R}^{n \times m}$, to minimize the mapping errors from $\boldsymbol{\Psi}$

$$
\mathbf{G}=\min _{\boldsymbol{\Phi}} \sum_{k=1}^{m}\left\|\mathbf{x}^{k}-\boldsymbol{\Phi} \boldsymbol{\Phi}^{\mathrm{T}} \mathbf{x}^{k}\right\|=\sum_{k=1}^{m}\left\|\mathbf{x}^{k}-\boldsymbol{\Psi} \boldsymbol{\Psi}^{\mathrm{T}} \mathbf{x}^{k}\right\|
$$

where $\boldsymbol{\Phi}$ is an arbitrarily chosen subspace which satisfies $\boldsymbol{\Phi}^{\mathrm{T}} \boldsymbol{\Phi}=\mathbf{I}$. The minimization problem is equivalent to

$$
\mathbf{H}=\max _{\boldsymbol{\Phi}} \sum_{k=1}^{m} \frac{\left\langle\left(\mathbf{x}^{k}, \boldsymbol{\Phi}\right)^{2}\right\rangle}{\|\boldsymbol{\Phi}\|^{2}}=\sum_{k=1}^{m} \frac{\left\langle\left(\mathbf{x}^{k}, \boldsymbol{\Psi}\right)^{2}\right\rangle}{\|\boldsymbol{\Psi}\|^{2}}
$$

where $(\cdot, \cdot)$ indicates an inner product operation, and $\langle\cdot\rangle$ indicates an averaging operation which can be omitted if the data ensemble is obtained from numerical simulation. The constraint optimization problem in Eq.(6) is transformed into the following Lagrange equation

$$
J(\boldsymbol{\Phi})=\sum_{k=1}^{m}\left(\mathbf{x}^{k}, \boldsymbol{\Phi}\right)^{2}-\lambda\left(\|\boldsymbol{\Phi}\|^{2}-1\right)
$$

where $\lambda$ is the Lagrange multiplier used to enforce the constraint on the norm of the basis vector. Solving the partial derivative of the objective function $\boldsymbol{J}(\boldsymbol{\Phi})$ with respect to $\boldsymbol{\Phi}$ gives

$$
\frac{d}{d \boldsymbol{\Phi}} J(\boldsymbol{\Phi})=2 \mathbf{X X}^{H} \boldsymbol{\Phi}-2 \lambda \boldsymbol{\Phi}
$$

where $\mathbf{X}=\left\{\mathbf{x}_{1}, \cdots, \mathbf{x}_{m}\right\}$ is the matrix of snapshots. By setting Eq.(8) to zero, the following equation is obtained

$$
\left(\mathbf{X X}^{\mathrm{T}}-\lambda \mathbf{I}\right) \boldsymbol{\Psi}=0
$$

Equation (9) is a real symmetry eigenvalue problem of the POD kernel, $\mathbf{K}=\mathbf{X} \mathbf{X}^{\mathrm{T}}$. For large systems, $\mathbf{K} \in \mathbf{R}^{n \times n}$ will be of a very large dimension, and the large eigenvalue problem is not easy to solve. Because $\mathbf{X} \mathbf{X}^{\mathrm{T}}$ and $\mathbf{X}^{\mathrm{T}} \mathbf{X}$ have the same eigenvalues, $\boldsymbol{\Psi}$ can also be calculated from the following lower $m$ dimensional problem 


$$
\left\{\begin{array}{l}
\mathbf{X}^{\mathrm{T}} \mathbf{X V}=\mathbf{V} \boldsymbol{\Lambda} \\
\boldsymbol{\Psi}=\mathbf{X V} \boldsymbol{\Lambda}^{-1 / 2}
\end{array}\right.
$$

where $\boldsymbol{\Psi}=\left(\Psi_{1}, \Psi_{2}, \cdots, \Psi_{m}\right), \boldsymbol{\Lambda}=\left(\lambda_{1}, \lambda_{2}, \cdots, \lambda_{\mathrm{m}}\right)$, and $\lambda_{1} \geq \lambda_{2} \geq \cdots \geq \lambda_{\mathrm{m}}$. The singular values are often referred to as the "energy" contained in the system. The number of retained POD basis vectors (POD modes), $r$, is determined by considering the energy captured with respect to the original model. The total captured energy level of an $r$-order subspace is defined as

$$
\eta(r)=\frac{\sum_{i=1}^{r} \lambda_{i}}{\sum_{i=1}^{m} \lambda_{i}}
$$

The number of POD modes retained should ensure capturing $99.99 \%$ of the energy in the original system. By truncating $\boldsymbol{\Psi}$ to the $r$-order vector $\boldsymbol{\Psi}_{r}=\left(\Psi_{1}, \Psi_{2}, \cdots, \Psi_{\mathrm{r}}\right)$, the fullorder series $\mathbf{x}^{n \times 1}$ can be reduced to an $r$-order system

$$
\mathbf{x}^{n \times 1}=\boldsymbol{\Psi}_{r} \xi^{r \times 1}
$$

\subsection{Model reduction for aeroelastic problem}

Consider the case of a CFD mesh with $n$ grid points: for a 2D problem, the total number of fluid degrees of freedom (DOFs) is $N_{f}=4 n$; and for a 3D problem, $N_{f}=5 n$. Generally, the number of grid points for a 3D mesh is several millions. To reduce the fluid problem to a manageable size, POD ROM technique (Hall et al., 2000; Lieu et al., 2005) is applied to the linearized fluid equations. To generate a state space model derived from the CFD equations, a converged steady-state CFD solution around the un-deformed (initial) geometry is computed, with the flow solution satisfying the relation $\mathbf{F}\left(\mathbf{w}_{0}, \mathbf{u}_{0}, \mathbf{u}_{c 0}\right)=0$, where $\dot{\mathbf{w}}_{0}=0, \mathbf{u}_{0}=0, \dot{\mathbf{u}}_{0}=0, \mathbf{u}_{c 0}=0$, $\dot{\mathbf{u}}_{c 0}=0$. Here, $\mathbf{u}_{c}$ denotes the control surface deflection. Then, the (nonlinear) Euler equations are linearized about the steady-state solution $\left(\mathbf{A}_{0}, \mathbf{w}_{0}, \mathbf{u}_{0}, \mathbf{u}_{\mathrm{c} 0}\right)$ through a first order Taylor's expansion. The FOM is formulated as

$$
\left\{\begin{array}{l}
\dot{\mathbf{w}}=\mathbf{A} \mathbf{w}+\mathbf{B} \mathbf{x}_{s}(t)+\mathbf{E} \mathbf{u}_{c}(t) \\
\mathbf{f}^{e x t}(t)-\mathbf{f}_{0}^{e x t}(t)=\mathbf{C w}
\end{array}\right.
$$

where

$$
\mathbf{A}=-\mathbf{A}_{0}^{-1} \frac{\partial \mathbf{F}}{\partial \mathbf{w}}
$$


$\mathbf{B}=-\mathbf{A}_{0}^{-1}\left[\frac{\partial \mathbf{F}}{\partial \mathbf{u}}, \quad \mathbf{w}_{0} \frac{\partial \mathbf{A}}{\partial \mathbf{u}}+\frac{\partial \mathbf{F}}{\partial \dot{\mathbf{u}}}\right]$

$\mathbf{C}=\frac{\partial \mathbf{f}^{e x t}}{\partial \mathbf{w}}$

$\mathbf{E}=-\mathbf{A}_{0}^{-1} \frac{\partial \mathbf{F}}{\partial \mathbf{u}_{c}}$

$\mathbf{x}_{s}(t)=[\mathbf{u}, \dot{\mathbf{u}}]^{\mathrm{T}}$

Then, the linearized fluid dynamic equations are projected on an $r$-order subspace, $\Psi_{r}=\left(\Psi_{1}, \Psi_{2}, \ldots, \Psi_{r}\right)$, generated from the POD method discussed above, with $r \ll N_{f}$. The interested reader may find more details on the linearized process and POD projection used in the present study in Refs. (Chen et al., 2010; Zhou et al., 2014). The system state $\mathbf{w}$ can be expressed as a modal expansion of the type

$$
\mathbf{w}^{N_{f} \times 1}=\boldsymbol{\Psi}_{r} \mathbf{r}_{r}^{r \times 1}
$$

where $\boldsymbol{\Psi}_{r} \in \mathbf{R}^{N_{f} \times r}$, and satisfying the relation $\boldsymbol{\Psi}_{r}^{\mathrm{T}} \boldsymbol{\Psi}_{r}=\mathbf{I}$. The low-order optimal orthonormal basis, $\boldsymbol{\Psi}_{r}$, is used to project the FOM in Eq.(13). The resulting ROM of the fluid system, with $r$ DOFs, is formulated as

$$
\left\{\begin{array}{l}
\dot{\mathbf{x}}_{r}=\mathbf{A}_{a} \cdot \mathbf{x}_{r}+\mathbf{B}_{a} \cdot \mathbf{x}_{s}(t)+\mathbf{E}_{a} \cdot \mathbf{u}_{c}(t) \\
\mathbf{f}^{e x t}(t)-\mathbf{f}_{0}^{e x t}(t)=\mathbf{C}_{a} \cdot \mathbf{w}
\end{array}\right.
$$

where $\mathbf{A}_{a}=\boldsymbol{\Psi}_{r}^{\mathrm{T}} \mathbf{A} \boldsymbol{\Psi}_{r}, \quad \mathbf{B}_{a}=\boldsymbol{\Psi}_{r}^{\mathrm{T}} \mathbf{B}, \mathbf{C}_{a}=\mathbf{C} \boldsymbol{\Psi}_{r}$, and $\mathbf{E}_{a}=\boldsymbol{\Psi}_{r}^{\mathrm{T}} \mathbf{E}$.

As the fluid and structural problems are physically characterised by different dynamics, in Eq.(15), $t$ indicates the fluid dimensional time which is related to $\tau$, the structural non-dimensional time, by $t=\tau \cdot \mathrm{V} / L_{r e f}=\tau / \omega$, with $L_{r e f}$ a characteristic length. Rewrite Eq.(15) in terms of structural time

$$
\left\{\begin{array}{l}
\dot{\mathbf{x}}_{a}(\tau)=\mathbf{A}_{a} \cdot \mathbf{x}_{a}(\tau) / \omega+\mathbf{B}_{a} \cdot[\mathbf{u}(\tau) / \omega, \dot{\mathbf{u}}(\tau)]^{\mathrm{T}}+\mathbf{E}_{a} \cdot \mathbf{u}_{c}(\tau) / \omega \\
\mathbf{f}^{e x t}(\tau)-\mathbf{f}_{0}^{e x t}(\tau)=\mathbf{C}_{a} \cdot \mathbf{x}_{a}(\tau)
\end{array}\right.
$$

By coupling Eqs.(4) and (16), the aero-servo-elastic equations in state space form are obtained

$$
\left\{\begin{array}{l}
\dot{\mathbf{x}}_{a s e}(\tau)=\mathbf{A}_{a s e} \cdot \mathbf{x}_{\text {ase }}(\tau)+\mathbf{B}_{\text {ase }} \cdot \mathbf{u}_{c}(\tau) \\
y_{\text {ase }}(\tau)=\mathbf{C}_{\text {ase }} \cdot \mathbf{x}_{\text {ase }}(\tau)
\end{array}\right.
$$

where

$\mathbf{x}_{\text {ase }}=\left[\mathbf{x}_{\mathrm{a}}, \mathbf{x}_{s}\right]^{\mathrm{T}}$ 


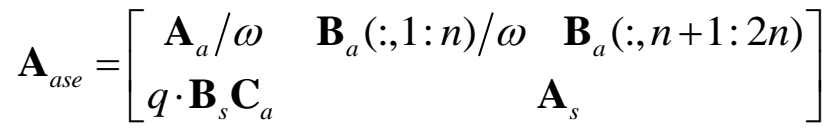

$$
\begin{aligned}
& \mathbf{B}_{\text {ase }}=\left[\begin{array}{c}
\mathbf{E}_{a} / \omega \\
\mathbf{0}
\end{array}\right] \\
& \mathbf{C}_{\text {ase }}=\left[\mathbf{0}, \mathbf{C}_{s}\right], y_{\text {ase }}=\mathbf{x}_{s}
\end{aligned}
$$

The total number of DOFs of the aero-servo-elastic system in Eq.(17) is $N_{d o f}=r+2 n$, which consists of $r$ POD modes and $n$ structural mode-shapes. The factor of 2 accounts for rewriting the structural problem in state space form.

\section{Control Design with an Input Time Delay}

In practice, it is supposed that an inherent constant time delay $t_{\mathrm{d}}$ is present in the control feedback loop. In Eq.(17), the control action is replaced by the delayed control action, $\mathbf{u}_{\mathrm{c}}\left(t-t_{\mathrm{d}}\right)$ :

$$
\left\{\begin{array}{l}
\dot{\mathbf{x}}_{\text {ase }}(t)=\mathbf{A}_{\text {ase }} \mathbf{x}_{\text {ase }}(t)+\mathbf{B}_{\text {ase }} \mathbf{u}_{c}\left(t-t_{d}\right)+\mathbf{E}_{a s e} w(t) \\
\mathbf{y}_{\text {ase }}(t)=\mathbf{C}_{\text {ase }} \mathbf{x}_{\text {ase }}(t)
\end{array}\right.
$$

where $w$ represents the disturbance input from atmospheric effects, i.e. atmospheric gust and turbulence (Tantaroudas and Da Ronch, 2015).

\subsection{Discrete-time System}

First, the continuous aero-servo-elastic ROM with a time delay is transformed into a delay-free equation in discrete form. Transform the continuous state-space form of Eq.(18) to a discrete form by integration between $t_{1}$ and $t_{2}$

$$
\mathbf{x}\left(\mathrm{t}_{2}\right)=\mathrm{e}^{\mathbf{A}_{a s e}\left(\mathrm{t}_{2}-\mathrm{t}_{1}\right)} \mathbf{x}\left(\mathrm{t}_{1}\right)+\int_{\mathrm{t}_{1}}^{\mathrm{t}_{2}} \mathrm{e}^{\mathbf{A}_{\text {ase }}\left(\mathrm{t}_{2}-t\right)}\left[\mathbf{B}_{\text {ase }} \mathbf{u}_{c}\left(t-t_{\mathrm{d}}\right)+\mathbf{E}_{\text {ase }} w(t)\right] \mathrm{d} t
$$

Let $t_{1}=k \Delta t, t_{2}=(k+1) \Delta t$. By replacing the integration variable $t$ with $\eta=(k+1) \Delta t-t$, Eq.(19) may be rewritten as

$$
\mathbf{x}[(k+1) \Delta t]=\mathrm{e}^{\mathbf{A}_{\text {ase }} \Delta t} \mathbf{x}(\mathrm{k} \Delta \mathrm{t})+\int_{0}^{\Delta t} e^{\mathbf{A}_{\text {ass }} \eta} \mathbf{B}_{\text {ase }} \mathbf{u}_{c}\left[(\mathrm{k}+1) \Delta \mathrm{t}-\mathrm{t}_{d}-\eta\right] \mathrm{d} \eta+\int_{0}^{\Delta t} e^{\mathbf{A} \eta} d \eta \mathbf{E}_{a s e} w(\mathrm{k} \Delta \mathrm{t})
$$

Given a sampling time step $\Delta t$, the time delay $t_{\mathrm{d}}$ can be expressed as (Hu and Wang, 2002)

$$
t_{d}=(l-m) \Delta t
$$


where $l$ is an integer (time step iteration) and $m$ satisfies $0 \leq m<1$. Equation(20) can be written as (see the derivation given in Appendix A)

$$
\mathbf{x}[(k+1)]=\mathbf{A}_{d} \mathbf{x}(k)+\mathbf{B}_{d 1} \mathbf{u}_{c}[k-l]+\mathbf{B}_{d 2} \mathbf{u}_{c}[k-l+1]+\mathbf{E}_{d} w(k)
$$

Introduce a new state space vector as

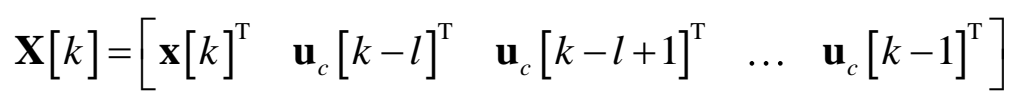

Then, the corresponding augmented state space equations are formulated as

$$
\mathbf{X}[k+1]=\mathbf{A X}[k]+\mathbf{B u}_{c}[k]+\mathbf{E} w[k]
$$

where

$$
\mathbf{A}=\left[\begin{array}{cccccc}
\mathbf{A}_{d} & \mathbf{B}_{d 1} & \mathbf{B}_{d 2} & 0 & \cdots & 0 \\
0 & 0 & \mathbf{I} & 0 & \cdots & 0 \\
0 & 0 & 0 & \mathbf{I} & \cdots & 0 \\
\vdots & \vdots & \vdots & \vdots & \ddots & 0 \\
0 & 0 & 0 & 0 & \cdots & \mathbf{I} \\
0 & 0 & 0 & 0 & \cdots & 0
\end{array}\right], \quad \mathbf{B}=\left[\begin{array}{c}
0 \\
0 \\
0 \\
\vdots \\
0 \\
\mathbf{I}
\end{array}\right], \mathbf{E}=\left[\begin{array}{c}
\mathbf{E}_{d} \\
0 \\
0 \\
\vdots \\
0 \\
0
\end{array}\right]
$$

\subsection{LQR Control Design}

A LQR method is used for the active control law design based on the aero-servoelastic ROM. The LQR assumes the availability of the entire state vector. In practice, some elements of the state vector may be unmeasurable, and suboptimal control is generally used, as in this work. The measured inputs are the displacements and velocities at wing tip where sensors are located. First, the LQR state feedback design method states that

$$
\mathbf{u}_{c}[k]=-\mathbf{K}^{*}\left(1: N_{d o f}\right) \cdot \mathbf{X}[k]
$$

The suboptimal control design method assumes that only a few linear combinations of system states can be directly measured from the sensors. Let $\mathbf{K}=\mathbf{K}^{*}\left(1: N_{d o f}\right) \cdot \mathbf{C}_{\text {ase }}^{\mathrm{T}} /\left(\mathbf{C}_{\text {ase }} \cdot \mathbf{C}_{\text {ase }}^{\mathrm{T}}\right)$. The output vector feedback control law is formulated

$$
\mathbf{u}_{c}[k]=-\mathbf{K} \cdot \mathbf{C}_{a s e} \cdot \mathbf{X}[k]=-\mathbf{K} \cdot y[k]
$$

For a conventional LQR method, the design problem for a delay-free system, see Eq.(17), aims at minimizing the cost function

$$
J_{0}=\sum_{k=0}^{\infty}\left\{\mathbf{x}^{\mathrm{T}}[k] \mathbf{Q}_{1} \mathbf{x}[k]+\mathbf{u}_{c}^{\mathrm{T}}[k] \mathbf{R u}_{c}[k]\right\}
$$


where $\mathbf{Q}_{1}$ is a semi-positive definite weighting symmetric matrix, and $\mathbf{R}$ is a positive definite weighting symmetric matrix. For the discrete-time state space model described in Eq.(24), a new performance index $\boldsymbol{J}$ is introduced

$$
J=\sum_{k=0}^{\infty}\left\{\mathbf{X}^{\mathrm{T}}[k] \mathbf{Q X}[k]+\mathbf{u}_{c}^{\mathrm{T}}[k] \mathbf{R} \mathbf{u}_{c}[k]\right\}
$$

where $\mathbf{Q}=\left[\begin{array}{ccccc}\mathbf{Q}_{1} & 0 & \cdots & 0 & 0 \\ 0 & 0 & \cdots & 0 & 0 \\ \vdots & \vdots & \ddots & \vdots & \vdots \\ 0 & 0 & \cdots & 0 & 0 \\ 0 & 0 & \cdots & 0 & 0\end{array}\right]$

The solution of the above optimization problem is:

$$
\begin{aligned}
& \mathbf{K}=\mathbf{R}^{-1} \mathbf{B P} \\
& \mathbf{P A}+\mathbf{A}^{\mathrm{T}} \mathbf{P}-\mathbf{P B R}^{-1} \mathbf{B}^{\mathrm{T}} \mathbf{P}+\mathbf{Q}=\mathbf{0}
\end{aligned}
$$

where $\mathbf{P}$ is the solution of the Riccati equation, which can be calculated by many iterative algorithms. Like the conventional LQR design method, the optimal state feedback gains were obtained in this work using MATLAB(R2011b)/SIMULINK.

\section{Test Cases}

\subsection{Pitch-plunge Aerofoil}

\subsubsection{Model Description}

The aerofoil section shown in Fig. 2 has two DoFs that define the motion about a reference axis (elastic axis, ea). The plunge deflection is denoted by $h$, positive downward, and $\alpha$ is the angle of attack about the elastic axis, positive with nose-up. The motion is restrained by two springs, $K_{h}$ and $K_{\alpha}$, which are representative of the bending and torsional stiffness, respectively. Structural damping is also included in both DoFs. A trailing-edge flap, with a chord equal to $25 \%$ of the aerofoil chord, is assumed massless and is used to control the aeroelastic vibrations and extend the stable flight region. The aerofoil semi-chord is denoted by $b$, the distance between the elastic axis and the centre of gravity is denoted by $x_{\alpha}$, and the sectional aerodynamic lift (positive upward) and moment (about the centre of gravity; positive with nose-up) by $\mathrm{L}$ and $\mathrm{M}$, respectively. 
For CFD calculations, an O-type 2D grid was generated which consists of 80 grid points along the aerofoil, and 30 grid points along the direction normal to the aerofoil surface, shown in Fig. 3. The total number of points is 2,400 and the number of DoFs of the FOM is 9,600. A preliminary study to guarantee grid-independent results was performed, and the current grid size and topology were deemed adequate for the flow conditions used.

A steady-state solution was computed at an angle of attack of zero degrees and Mach number of 0.875 . For the ROM generation, 50 POD modes were extracted from the snapshot matrix. Compared to the FOM which consists of 9,600 DoFs, the ROM has 50 DoFs and was found adequate to model the system dynamics, as shown in the sections to follow.

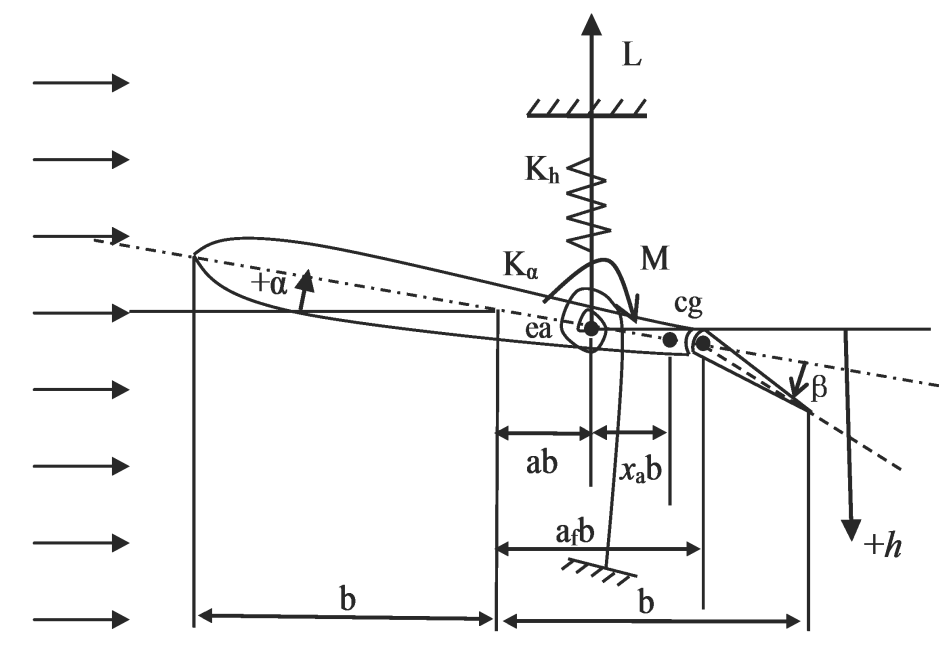

Fig. 2 Schematics of a 2D aerofoil section with trailing-edge control surface

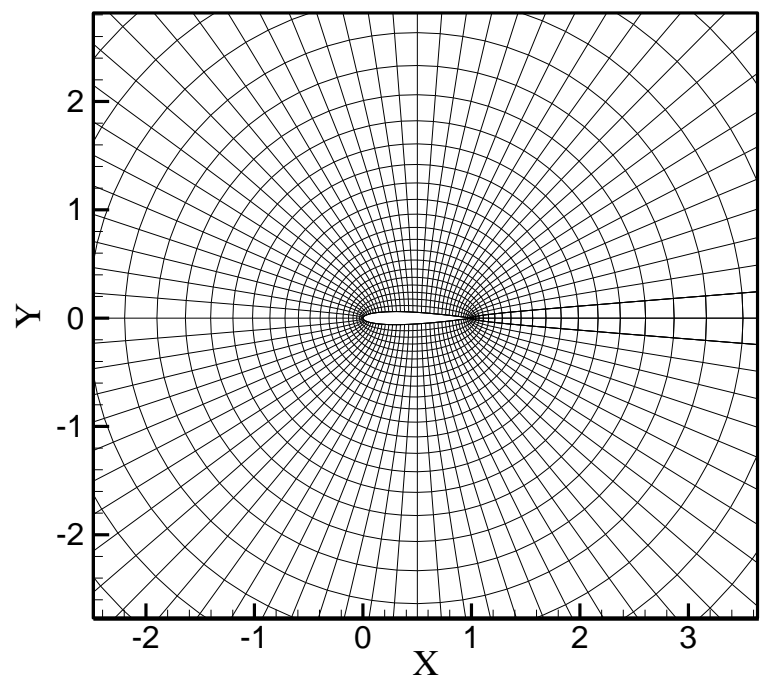


Fig. 3 Computational domain of the 2D aerofoil section

\subsubsection{Open-loop Flutter Boundary}

For the validation of the ROM, the test case is for the transonic flutter boundary of the NACA 64A010 aerofoil. Multiple flutter points in transonic conditions were found, as reported for example in (Alonso and Jameson, 1994; Feng et al., 2001; Huang et al., 2015a; Timme and Badcock, 2009). The aeroelastic model parameters are summarised in Table $\mathbf{1 .}$

Table 1. Aeroelastic parameters of the NACA64A010 aerofoil

\begin{tabular}{cccccc}
\hline Parameter & $\omega_{\mathrm{h}} / \omega_{\alpha}$ & $\mu$ & $a$ & $x_{\alpha}$ & $r_{\alpha}^{2}$ \\
\hline Value & 3.48 & 60 & -2.0 & 1.8 & 3.48 \\
\hline
\end{tabular}

For flutter analysis, the non-dimensional parameter VF is used

$$
\mathrm{VF}=\frac{V_{\infty}}{\left(\omega_{\alpha} \mathrm{b}\right) \sqrt{\mu}}
$$

According to Lyapunov's second method of stability, the open-loop aeroelastic system in Eq.(17) is asymptotically stable if all real parts of the eigenvalues of the system matrix, $\mathbf{A}_{\text {ase }}$, are negative. Stability can be deduced from the tracing of the eigenvalue with the largest real part, $\max (\operatorname{Re}(\lambda))$, for increasing flow speed or dynamic pressure. If $\max (\operatorname{Re}(\lambda))<0$, the open-loop aeroelastic system is asymptotically stable. The critical flutter point is identified when $\max (\operatorname{Re}(\lambda))$ becomes positive. Fig. 4 shows the variation of $\max (\operatorname{Re}(\lambda))$ with the non-dimensional parameter VF as computed by the ROM at Mach number of 0.875 . The ROM correctly identifies three neutrally stable points $\left(\mathrm{VF}_{1}=0.612, \mathrm{VF}_{2}=1.730, \mathrm{VF}_{3}=\right.$ 2.343), which are all in good agreement with (Timme and Badcock, 2009) that has three neutrally stable points $\left(\mathrm{VF}_{1}=0.568, \mathrm{VF}_{2}=1.87, \mathrm{VF}_{3}=2.50\right)$ at this condition. 


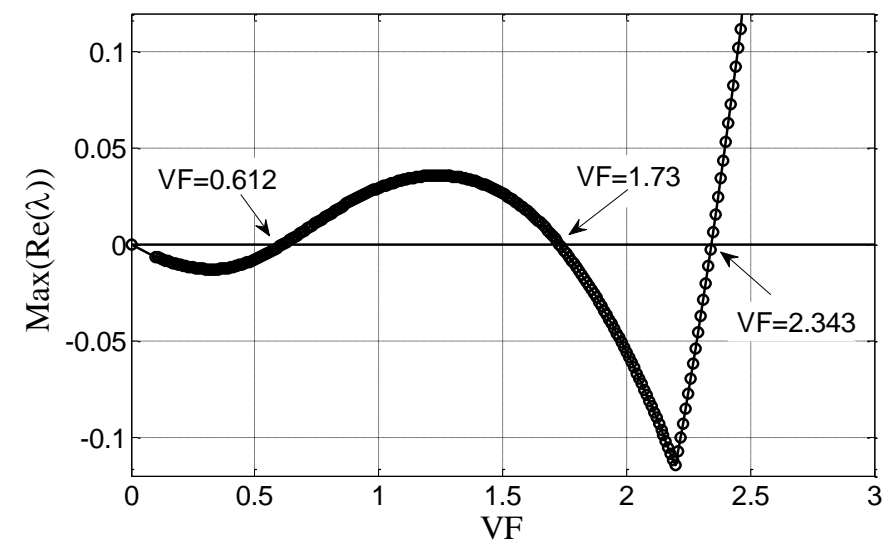

Fig. 4 Dependency of the system's eigenvalue with the largest real part on VF (NACA64A010 aerofoil, $\mathrm{M}=0.875$ )

The aeroelastic response of the pitch-plunge aerofoil section at the three flutter points is illustrated in Fig. 5. The dynamic responses were computed using the ROM, which is found adequate to predict the neutral stability (harmonic motion without damping) without adding or removing spurious energy. Once the ROM is generated, the search for the flutter points and the aeroelastic dynamic responses were obtained on the orders of seconds. This demonstrates that the ROM has the capability to accurately predict the system properties at a fraction of the time required for the FOM flutter assessment.
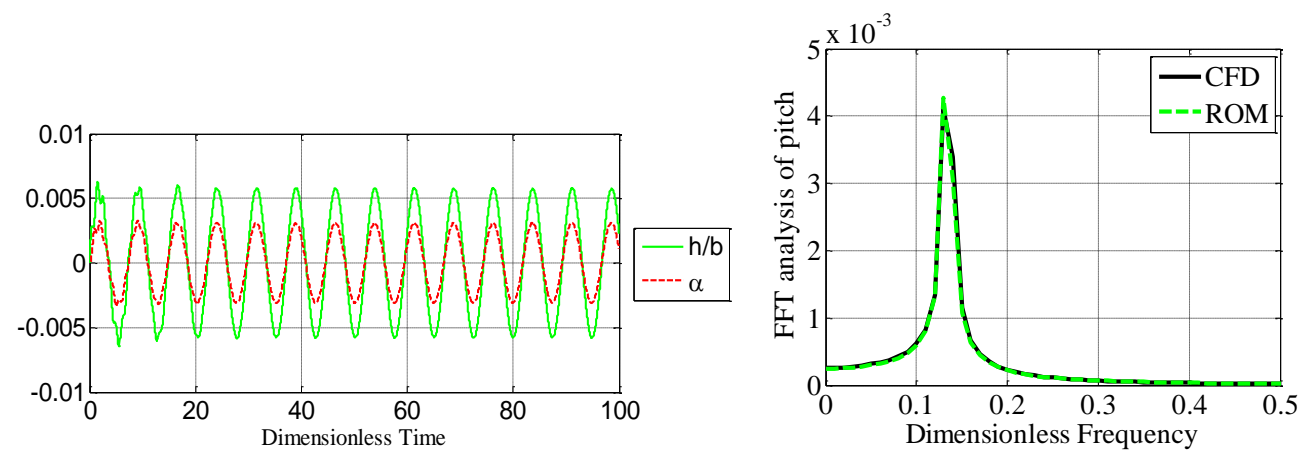

(a) $\mathrm{VF}_{1}=0.612$
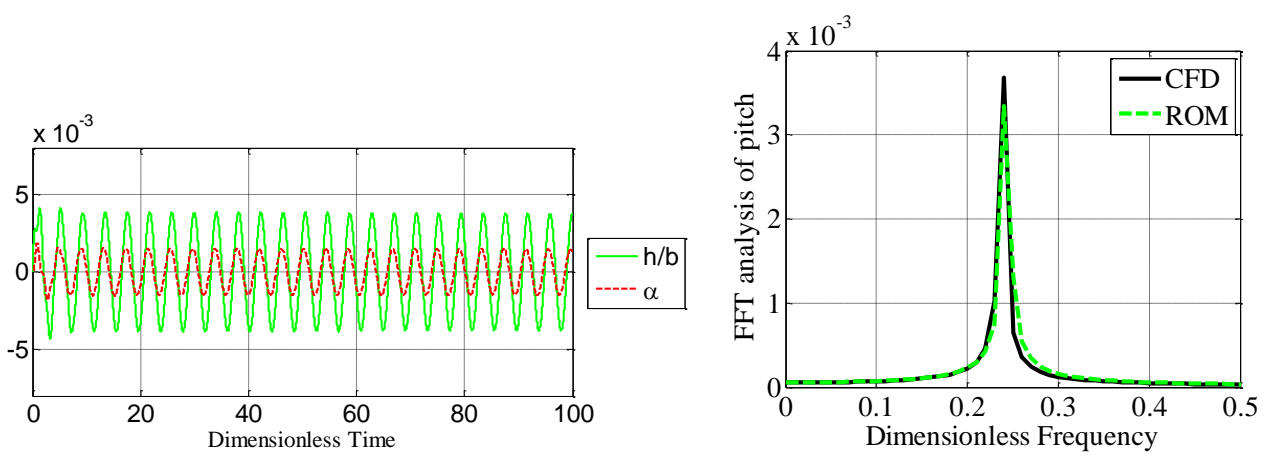

(b) $\mathrm{VF}_{2}=1.730$ 

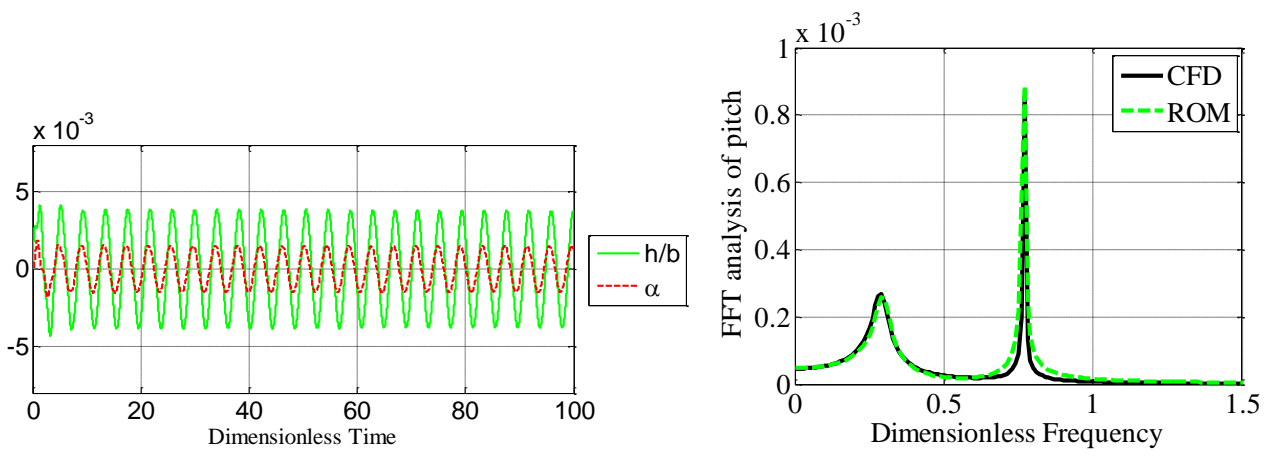

(c) $\mathrm{VF}_{3}=2.343$

Fig. 5 Response in pitch and plunge computed by ROM at three neutrally stable points (left), frequency content from the coupled CFD/CSD model and the ROM (right), (NACA64A010 aerofoil, $\mathrm{M}=0.875$ )

To further demonstrate the accuracy of the open-loop aeroelastic predictions for flutter analysis, Fig. 6 shows the flutter boundary of the NACA64A010 aerofoil. It is found that not only the FOM achieves a good agreement with available data (Alonso and Jameson, 1994; Feng et al., 2001), but also that the ROM captures the main features of the $S$-shaped flutter boundary.

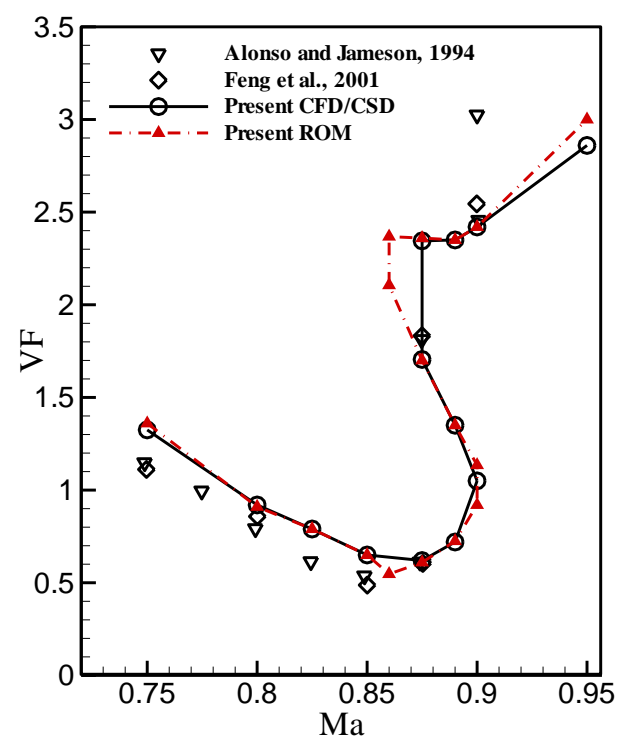

Fig. 6 Flutter boundary of NACA 64A010 aeroelastic system

\subsection{Three-dimensional Wing}

\subsubsection{Model Description}

The 3D test case is for the AGARD 445.6 wing (Yates et al., 1963; Yates Jr, 1988). The leading-edge sweep angle is $45 \mathrm{deg}$, the aerofoil section is the NACA 
$65 \mathrm{~A} 004$, and the wing has an aspect ratio of 1.65 and taper ratio of 0.6576 . For the fluid model, a multi-block structured mesh of about 0.2 million grid points is used. The grid, shown in Fig. 7(a), has the far-field located at 9 wing chords, with boundary conditions of solid, far-field, and symmetry plane set on appropriate boundary zones of the grid. A grid convergence study was carried out, and the dynamic response of the first four modal coordinates is reported in Fig. 8. Initial conditions were set for the velocity of the first modal coordinate. The medium grid was found adequate to predict the dynamic response. The total number of DoFs for the FOM is about one million.
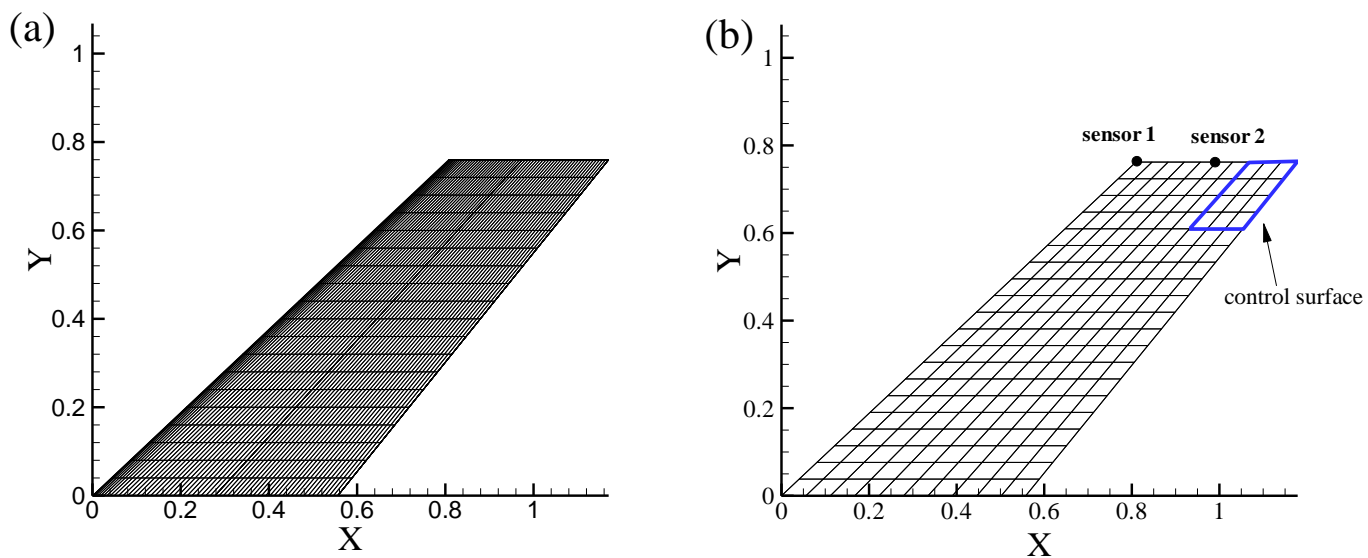

Fig. 7 Meshes for CFD and CSD solver: (a) Surface CFD grid of the AGARD 445.6 wing (left); and (b) structural FEM and trailing-edge control surface of the AGARD 445.6 wing (right)

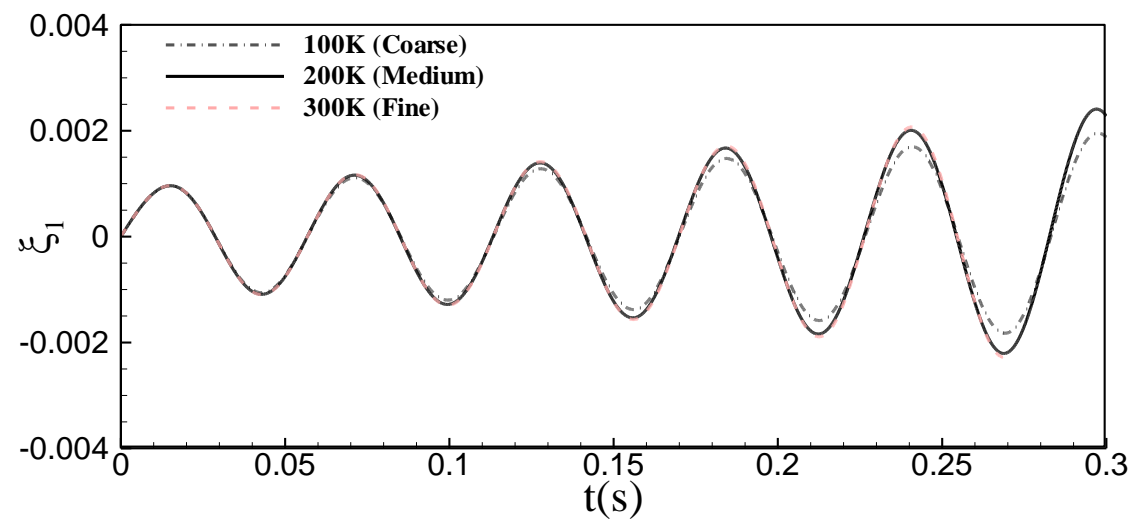



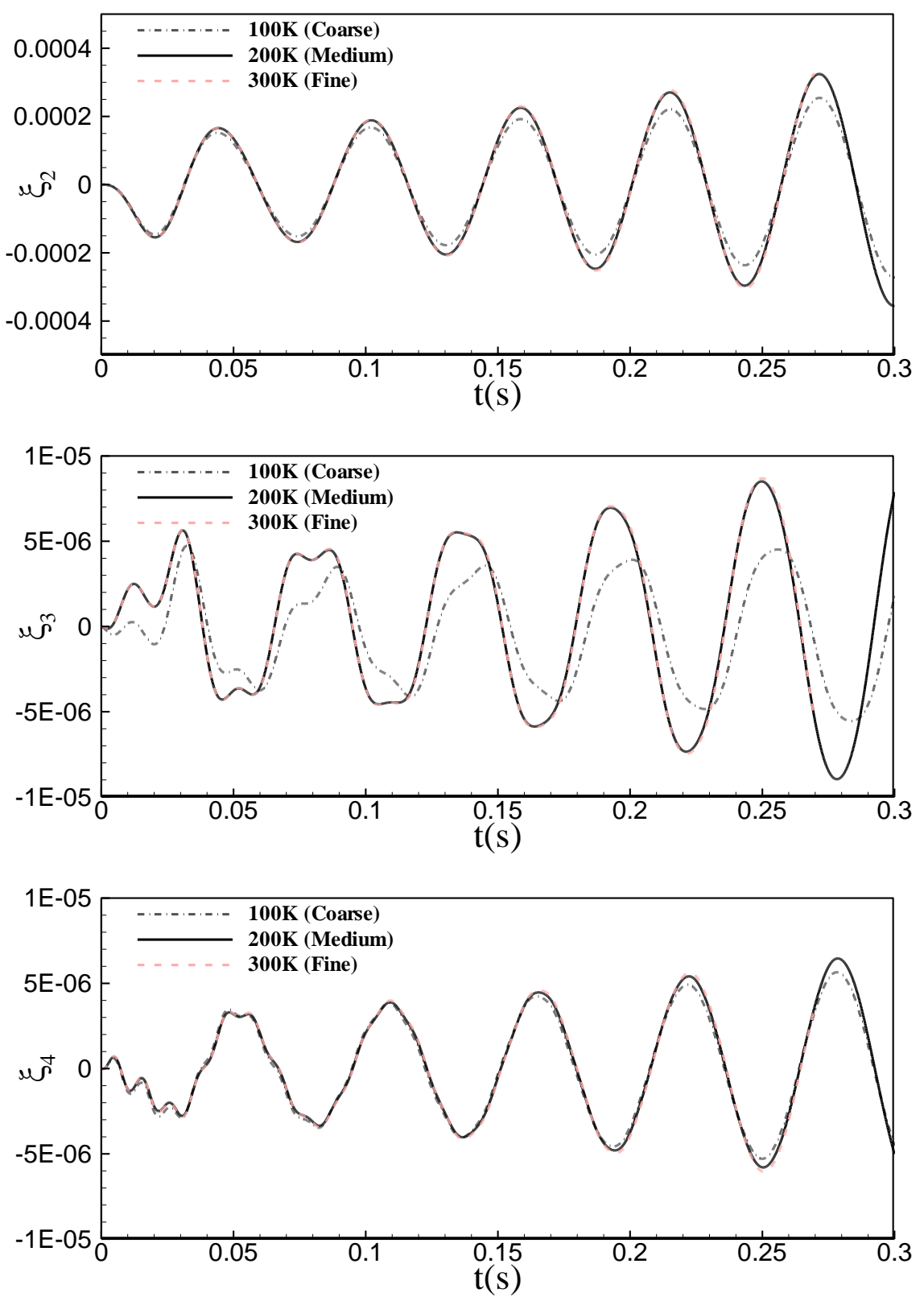

Fig. 8 Aeroelastic responses of the first four modal coordinates for the AGARD 445.6 wing for increasing number of grid points in the CFD mesh. $\left(\mathrm{M}=0.901, \mathrm{AOA}=0^{\circ}, \mathrm{VF}=310 \mathrm{~m} / \mathrm{s}\right.$;

$$
\text { flutter VF }=288.4 \mathrm{~m} / \mathrm{s} \text { ) }
$$

The aerodynamic and structural models of the AGARD 445.6 wing were modified to accommodate a trailing-edge control surface, located at the wing tip, to suppress aeroelastic instabilities. The control surface, with dimensions equal to $20 \%$ of the wing span and 30\% of the wing chord, is shown in Fig. 7(b). Two sensors are added at the wing tip to measure the displacements and velocities. For the structure, the modal decomposition is used. The wing structural dynamics are modelled using the first four elastic modes, with frequencies $\mathrm{f}_{1}=9.46 \mathrm{~Hz}, \mathrm{f}_{2}=39.71 \mathrm{~Hz}, \mathrm{f}_{3}=49.51$ 
$\mathrm{Hz}$, and $\mathrm{f}_{4}=95.13 \mathrm{~Hz}$. The motion of the control surface is treated as an additional mode-shape. Fig. 9 shows the mode-shape for the control surface deflection on the CFD surface. Rotations of the control surface are small, and modelling gaps were neglected.

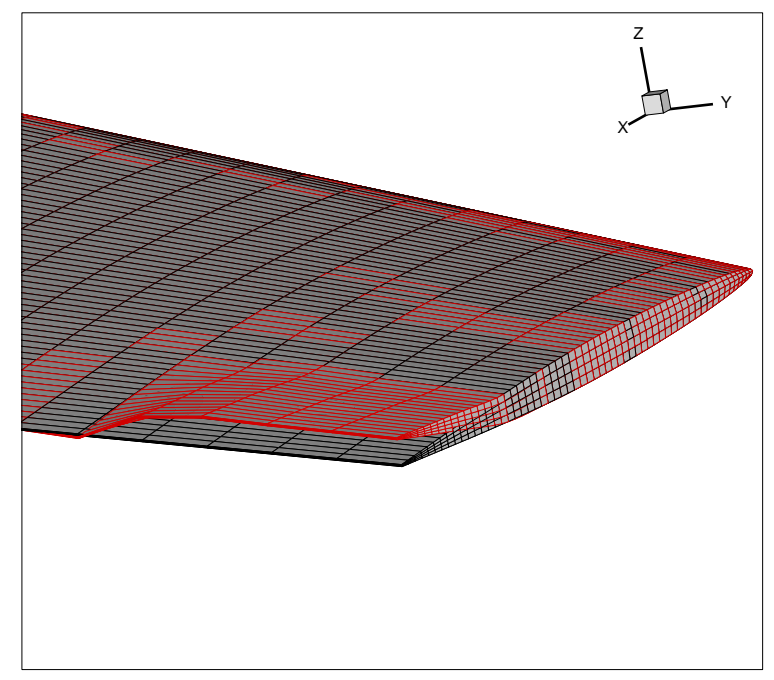

Fig. 9 The mode-shape for the control surface rotation on the CFD surface (in red)

\subsubsection{Open-loop Flutter Boundary}

The flutter boundary of the wing is analysed at $\mathrm{M}=0.678,0.901,0.960$ and zero angle of attack. The ROM was generated in two steps. The POD was used to create a first ROM with 600 DoFs. As the size was inadequate to design the control law, the BT method (Chen et al., 2012b) was then used to reduce the size of the fluid ROM to 50 states. The resulting aero-servo-elastic model consists of 60 DoFs: 50 from the POD/BT process, and $5+5$ for the structural modal displacements and velocities. Table 2 summarises the computational costs of the ROM compared to the FOM CFD/CSD analysis. CPU times are normalised by the cost of a steady-state CFD solution (90 minutes shown in Fig. 10) converged by 7 orders of magnitude. For the ROM generation: $a$ ) the linearization of the Euler equations is an inexpensive step; $b$ ) the computation of the snapshots is performed in much less than three steady-state CFD runs (2.40, to be precise) because of the slower convergence rates of the GMRES linear solves (Amsallem, 2010) in the FOM; and $c$ ) the calculation of the POD basis for the projection is equivalent to about three steady-state CFD runs as this requires performing the singular value decomposition (SVD) of a large-scale snapshot matrix and the construction of the POD basis. For a quantitative comparison, an 
unsteady time-domain CFD solution obtained with standard settings (time step of $0.0001 \mathrm{~s}, 100$ iterations per time step, and total simulation time of $0.5 \mathrm{~s}$ ) is equivalent to about 30 steady-state CFD runs, see Fig. 11. On the other hand, the generation of the ROM, including the initial steady-state CFD solution, is equivalent to about 6 steady-state solutions. Once generated, the ROM not only provides predictions for the flutter boundary and dynamic aeroelastic response at no extra cost, but allows designing practically a controller for flutter suppression.

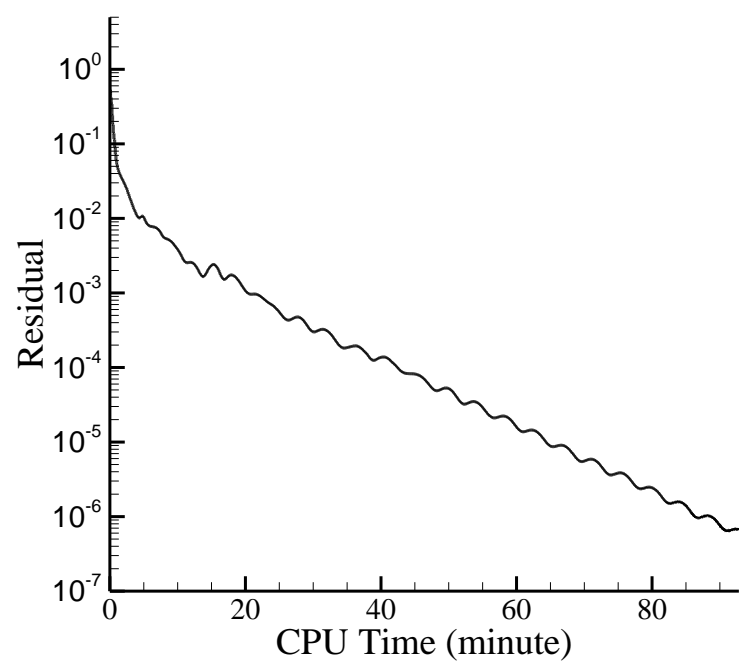

Fig. 10 Residual versus CPU time for a steady-state CFD solution, (AGARD 445.6 wing, $\mathrm{M}=0.901, \mathrm{AOA}=0^{\circ}$ )

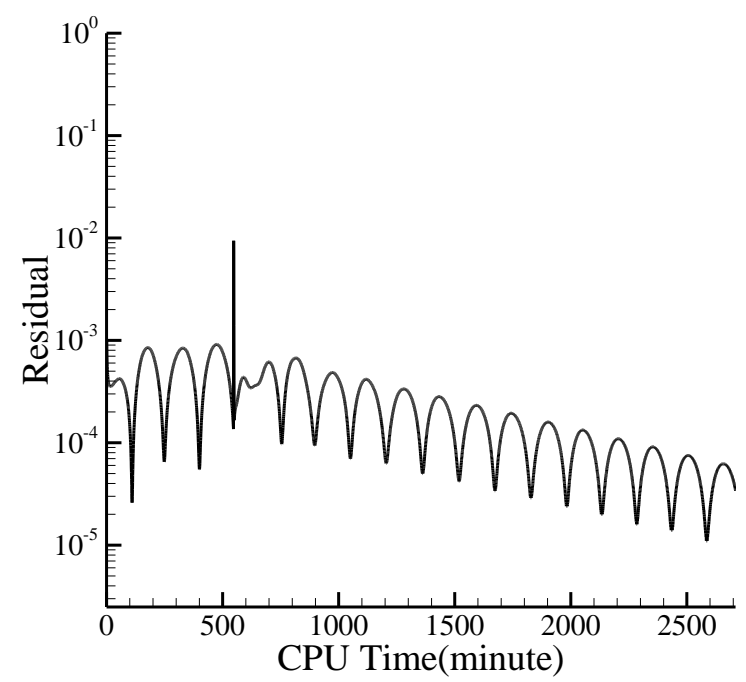

Fig. 11 Residual versus CPU time for an unsteady time-domain CFD solution (AGARD 445.6 wing, $\mathrm{M}=0.901, \mathrm{AOA}=0^{\circ}, \mathrm{VF}=310$ ) 
Table 2. Cost of ROM generation; reference cost for a steady-state CFD solution is about 90 min on Intel(R) Core(TM) i7-2600 CPU with $3.40 \mathrm{GHz}$

CPU time (Normalized by the cost

of the steady-state solution)

\begin{tabular}{cc}
\hline Steady-state Euler solution & 1.00 \\
Linearized nonlinear Euler (FOM) & 0.01 \\
$225 * 5$ snapshots generation & 2.40 \\
POD basis extraction and projection & 3.00 \\
$(\mathrm{ROM})$ & \\
\hline Total CPU time & 6.41 \\
\hline
\end{tabular}

The critical flutter point at a given condition can be efficiently determined by the stability method, as described for the 2D test case. Fig. 12 (a)-(c) show the dependency of the system eigenvalue with the largest real part, $\max (\operatorname{Re}(\lambda))$, on VF at three Mach numbers $(0.678,0.901$, and 0.960). At a given flight condition (Mach and angle of attack), the search for the flutter points is executed in order of seconds using the efficient ROM.

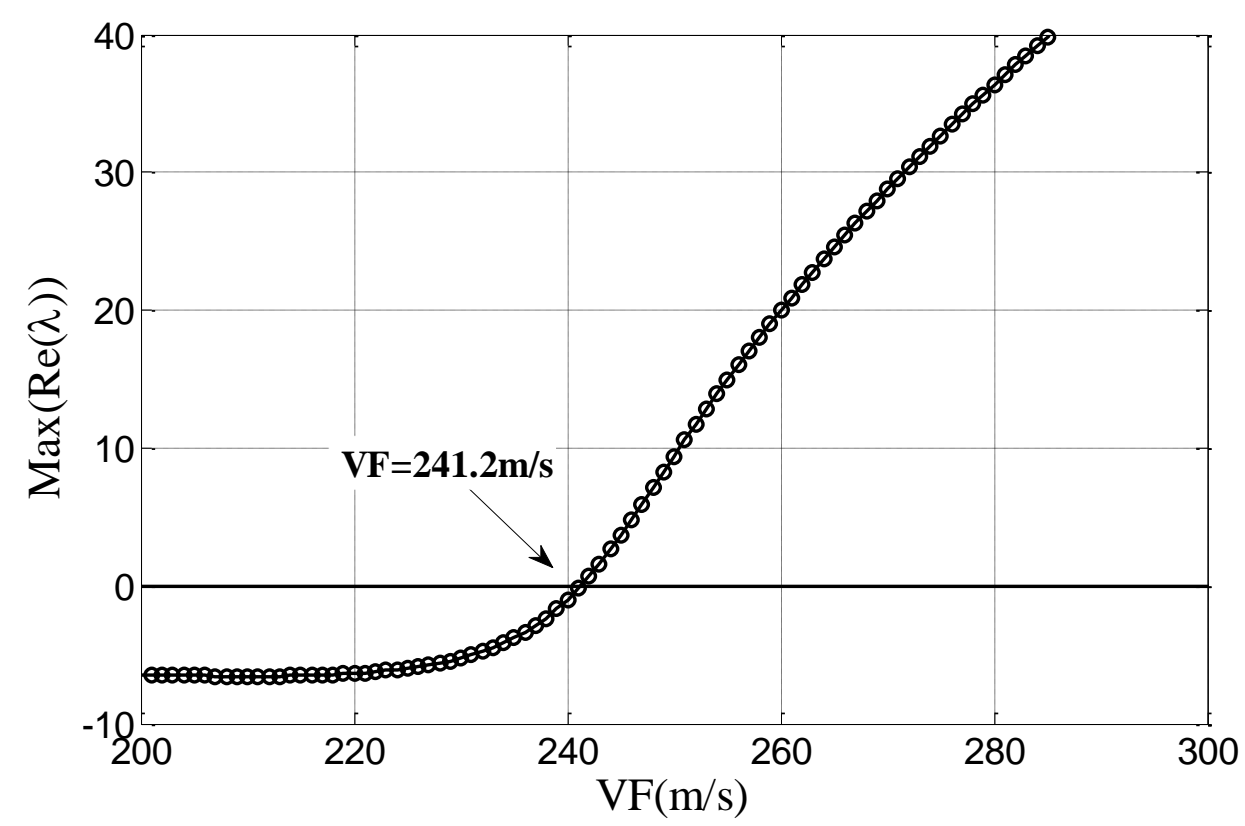

(a) $\mathrm{M}=0.678$ 


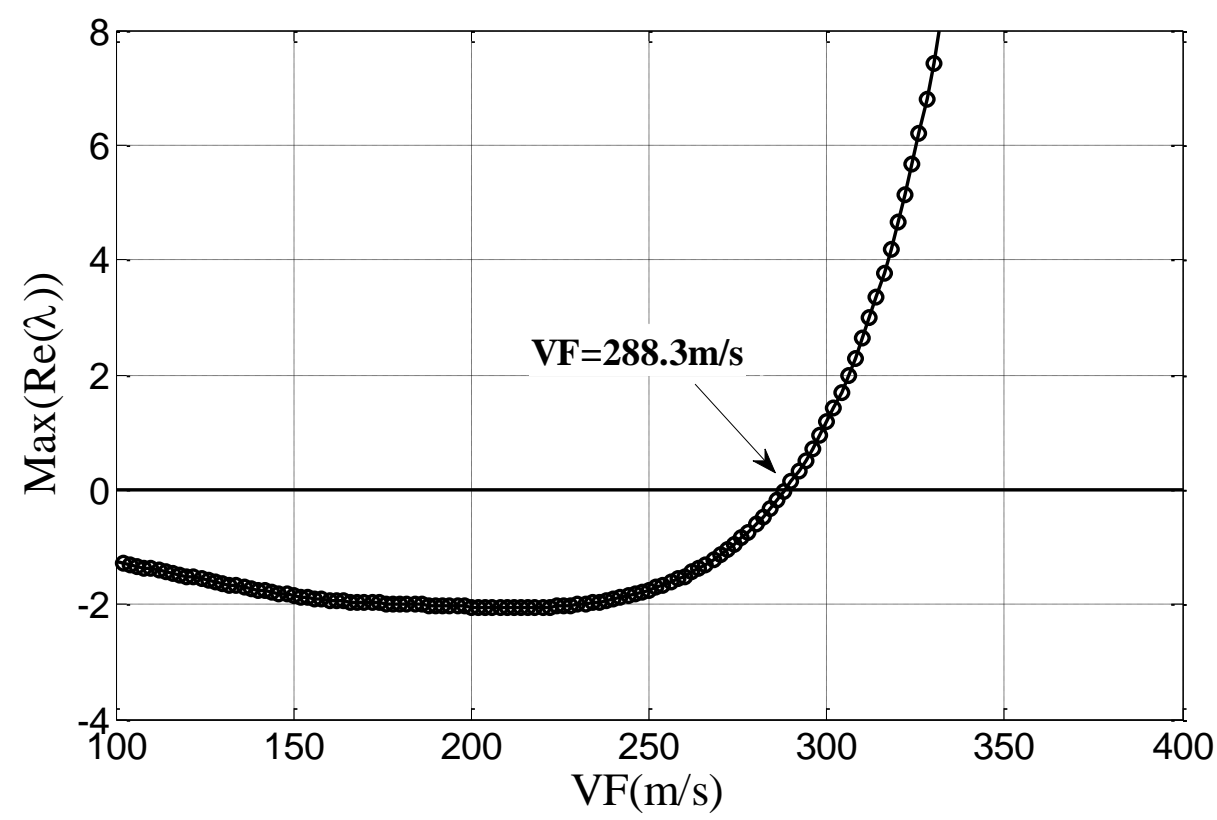

(b) $\mathrm{M}=0.901$

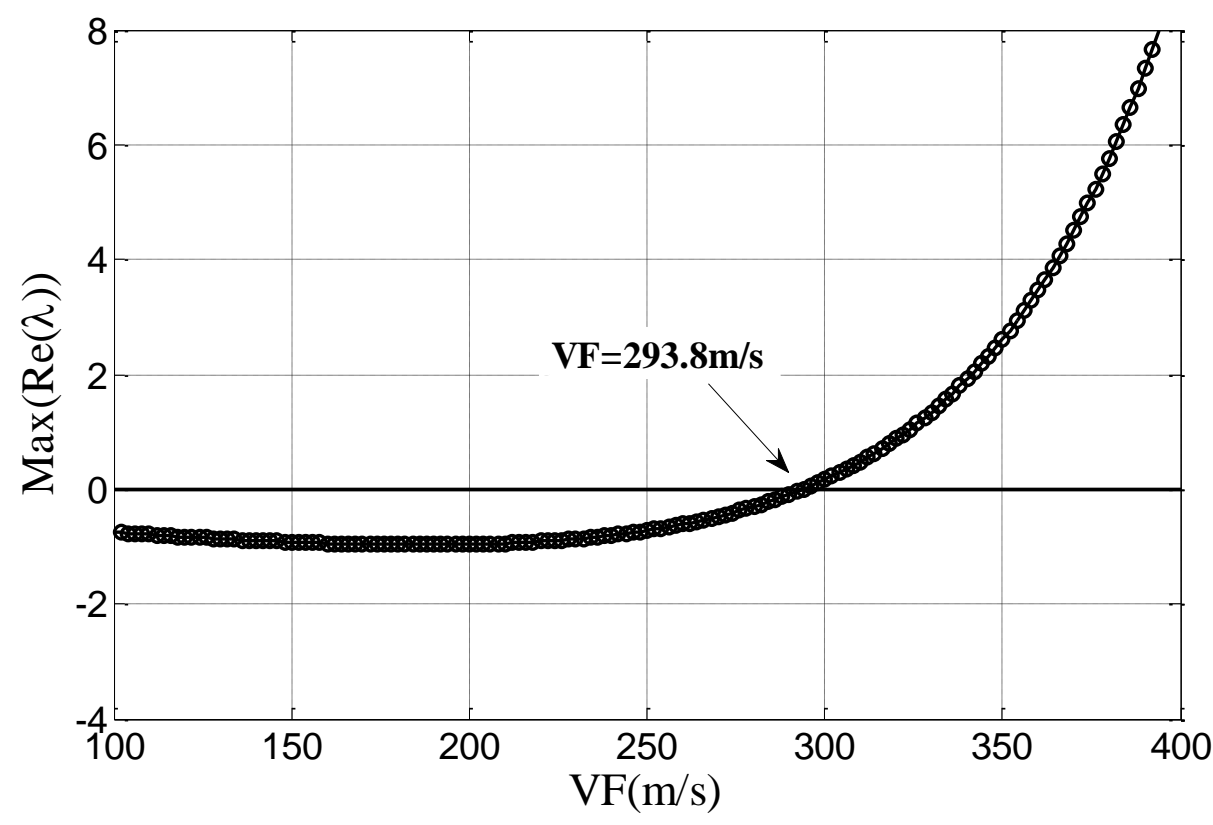

(c) $\mathrm{M}=0.960$

Fig. 12 Dependency of the system eigenvalue with the largest real part on VF (AGARD 445.6 wing)

The flutter boundary for the AGARD 445.6 wing is shown in Fig. 13. Experimental data are taken from (Yates Jr, 1988). The fully coupled CFD/CSD method predicts well the flutter boundary, from both a quantitative and qualitative standpoint. This ensures that the baseline FOM approach and the aerodynamic/structural models are adequate in capturing the relevant coupled 
nonlinear phenomena. It is also found that the aeroelastic ROM achieves an excellent agreement with the FOM, validating the ROM generation process and the ROM predictive capabilities for transonic flow conditions.

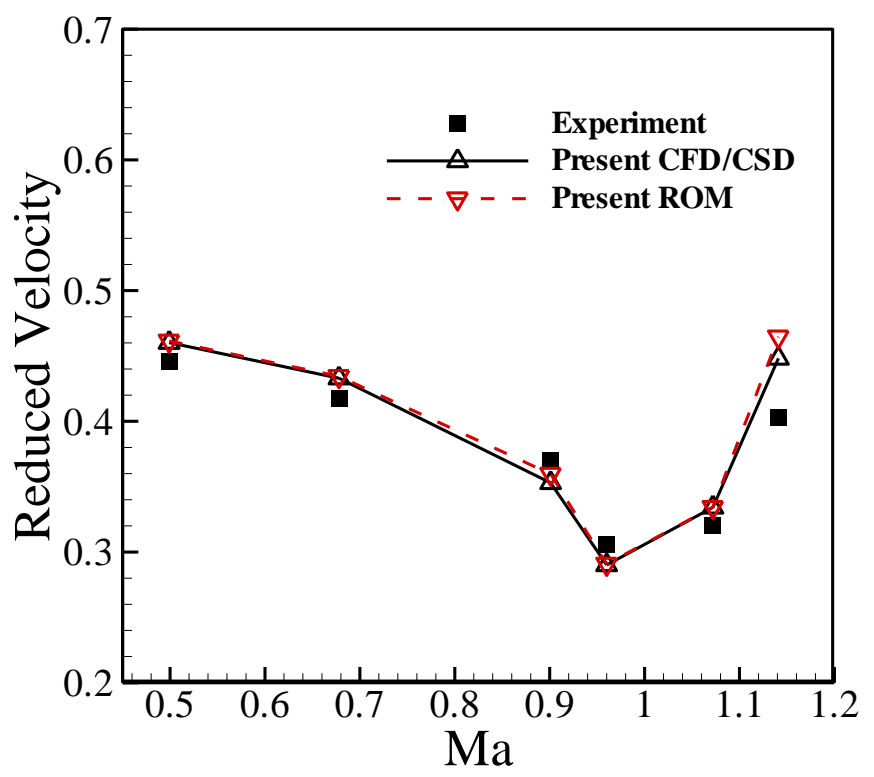

Fig. 13 Flutter boundary of the AGARD 445.6 wing; experimental data from (Yates Jr, 1988)

\section{Results}

\subsection{Pitch-plunge Aerofoil}

\subsubsection{Active Flutter Suppression}

Having validated the ROM generation and its general predictive capabilities for flutter boundary identification and dynamic aeroelastic response, the active flutter suppression is presented in this section. The test case is for a pitch-plunge NACA0012 aerofoil section (Badcock et al., 2004) which exhibits one single flutter point at transonic speed. The aerofoil section and the aeroelastic parameters differ from those presented in Section 4.1, and are summarized in Table 3 for the present test case. The authors carried out a systematic analysis for this problem, see (Zhou et al., 2014) and (Da Ronch et al., 2012), making it a suitable problem for control design synthesis.

Table 3. Aeroelastic parameters of the NACA0012 aerofoil

\begin{tabular}{cccccc}
\hline Parameter & $\omega_{\mathrm{h}} / \omega_{\alpha}$ & $\mu$ & $a$ & $x_{\alpha}$ & $r_{\alpha}^{2}$ \\
\hline Value & 0.342 & 100.0 & -0.2 & 0.2 & 0.539 \\
\hline
\end{tabular}


The trailing-edge control rotation is denoted by $\beta$, and is determined from an output feedback action

$$
\beta\left(t-t_{d}\right)=-\mathbf{K} \cdot \mathbf{y}_{\text {ase }}\left(t-t_{d}\right)=-\left[k_{1}, k_{2}, k_{3}, k_{4}\right] \cdot[h / b, \alpha, \dot{h} / b, \dot{\alpha}]^{\mathrm{T}}
$$

The control gains are computed using the LQR method for a delay-free aero-servoelastic ROM, i.e. $t_{\mathrm{d}}=0$. The feedback controller is designed at $\mathrm{M}=0.8$ and $\mathrm{VF}=3.8$, which is well above the flutter speed $(\mathrm{VF}=3.23)$. For a delay-free system, the control gains are found to be $k_{1}=0.1461, k_{2}=0.1089, k_{3}=-2.1868$, and $k_{4}=1.4228$. To demonstrate the adequacy of the feedback controller designed using the ROM, the control law is implemented in the coupled CFD/CSD model. The controller is switched on at $t=0.1838 \mathrm{~s}$ within the simulation to allow unstable aeroelastic vibrations to grow before activating the closed-loop suppression. Fig. 14 shows the closed-loop aeroelastic response computed by the FOM for various values of the time delay. Active flutter suppression is achieved for the delay-free system. It is not unexpected that the performance of the active feedback controller decreases for increasing time delay. For cases with a time delay smaller than $t_{\mathrm{d}}=0.0092 \mathrm{~s}$, flutter suppression is achieved with a degraded performance. For a time delay of $t_{\mathrm{d}}=0.0092 \mathrm{~s}$, the controller fails to suppress completely the aeroelastic vibrations, and the openloop instability turns into a motion with constant amplitudes. For the larger time delay, the feedback controller designed on a delay-free system is unable to suppress or control the unstable oscillatory motion.

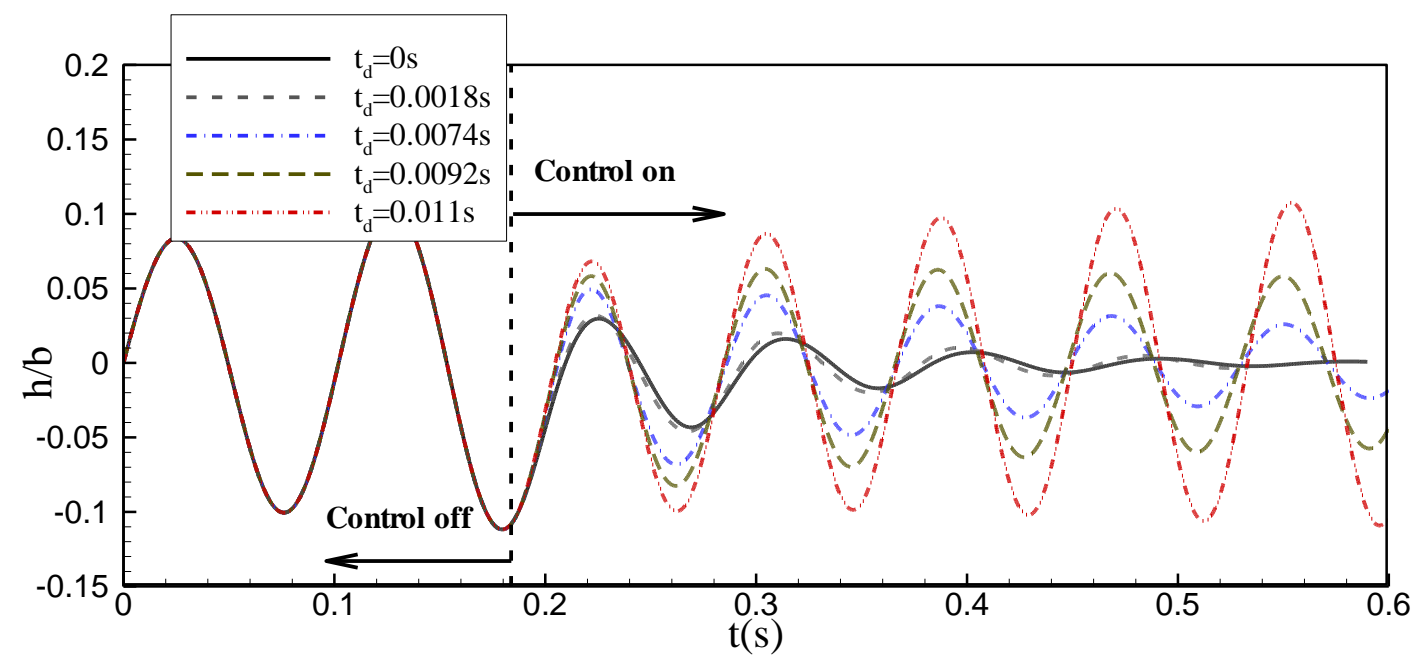




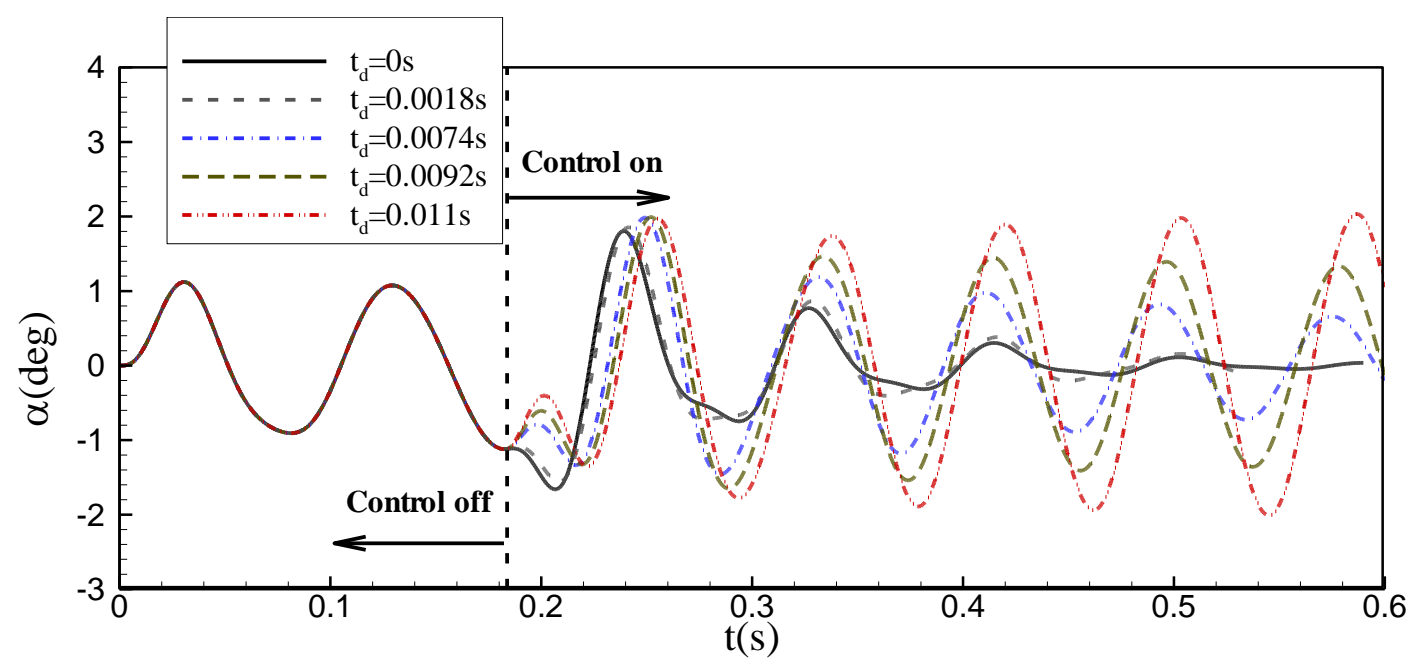

Fig. 14 Closed-loop response computed by the coupled CFD/CSD method for various control input time delays; feedback controller designed on nominal (delay-free) plant (NACA0012, $\mathrm{M}=0.8, \mathrm{VF}=3.8$; flutter $\mathrm{VF}=3.23$ )

\subsubsection{Active Flutter Suppression with Time-delay}

The time delay occurring between the measurement and the control action has a great effect on the controller effectiveness to suppress flutter. Three cases with a time delay larger than the time delay causing the controller to lose its effectiveness, see Section 5.1.1, are used here. For each case, a controller was designed based on Eq.(24) accounting for the known time delay. Table 4 summarises the three time delays and the corresponding control gains.

Table 4. Gains of LQR controller designed for different time delays

\begin{tabular}{ccccc}
\hline & $k_{1}$ & $k_{2}$ & $k_{3}$ & $k_{4}$ \\
\hline$t_{d}=0.0000 s$ & 0.1461 & 0.1089 & -2.1868 & 1.4228 \\
$t_{d}=0.0092 s$ & 0.2767 & 0.2377 & -0.0369 & 2.9253 \\
$t_{d}=0.0184 s$ & 0.3351 & -0.2210 & 2.2752 & 3.2244 \\
$t_{d}=0.0276 s$ & 0.2528 & -0.5072 & 4.1732 & 1.2167
\end{tabular}

Fig. 15 shows the aeroelastic response from the CFD/CSD model. The controllers are switched on at $t=0.1838 \mathrm{~s}$ within the simulation to allow unstable aeroelastic vibrations to grow before activating the closed-loop suppression. Oscillatory motions decay quickly to zero for all cases. This shows that the controllers designed from the time delay ROMs achieve flutter suppression. It is worth noting that, although the responses for different time delays are similar, control gains are significantly different, as reported in Table 4. 

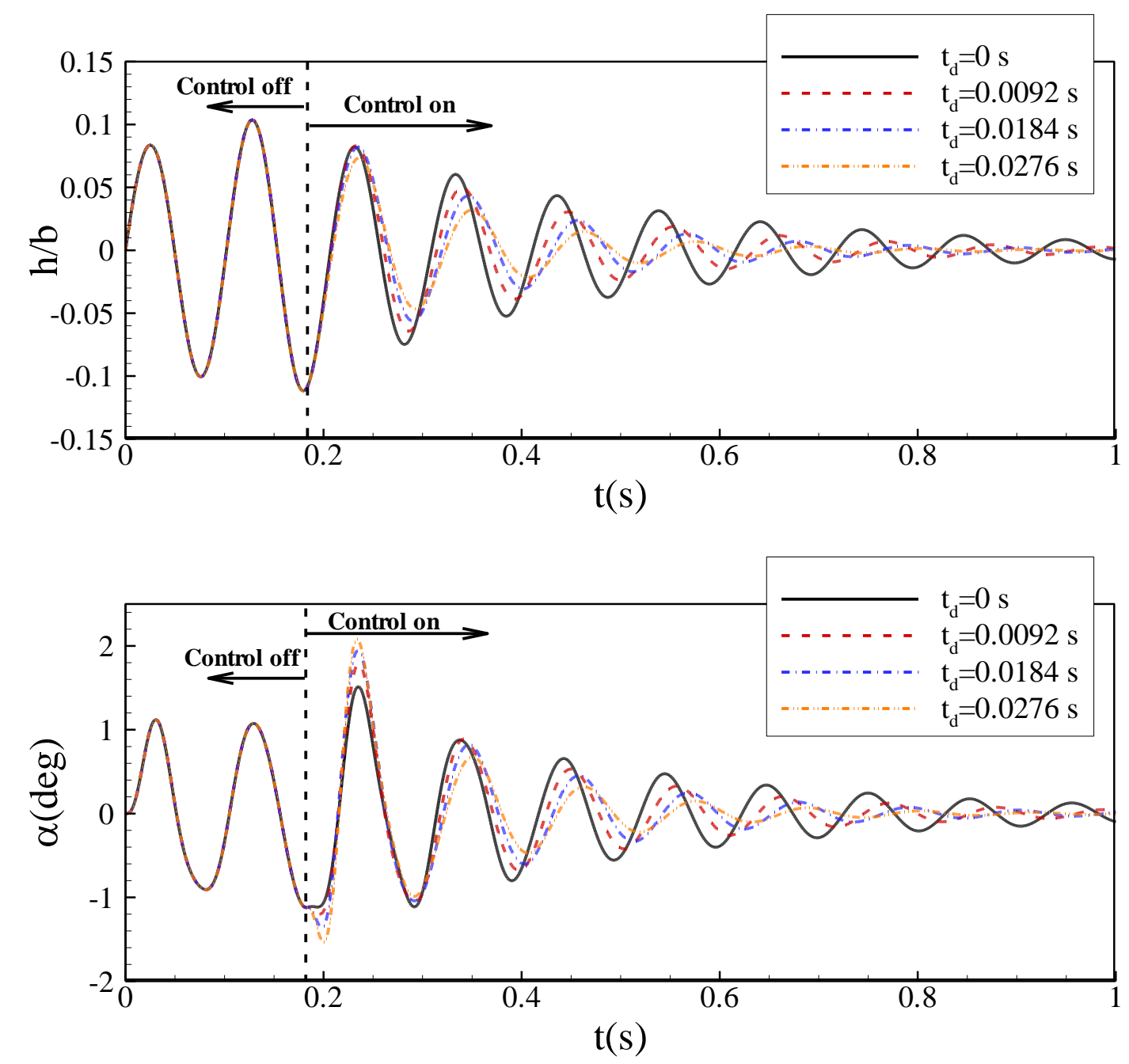

Fig. 15 Closed-loop response computed by the coupled CFD/CSD method for various control input time delays; feedback controller designed on actual (time-delayed) plant (NACA0012, $\mathrm{M}=0.8, \mathrm{VF}=3.8 ;$ flutter $\mathrm{VF}=3.23$ )

\subsection{Three-dimensional Wing}

\subsubsection{Active Flutter Suppression}

For the AGARD 445.6 wing, the freestream speed is set to $\mathrm{V}=310 \mathrm{~m} / \mathrm{s}$, which is above the flutter speed VF $=288.4 \mathrm{~m} / \mathrm{s}$ at Mach number $\mathrm{M}=0.901$. Previous research (Chen et al., 2012a) showed that an active controller can extend the AGARD 445.6 wing flutter boundary by about 20-30\%. As a representative flow condition, a speed $8 \%$ above the flutter speed is chosen here. The aero-servo-elastic ROM derived from the $\mathrm{POD} / \mathrm{BT}$ process is used to design the feedback controller, initially for a delayfree system. The weight matrices, $\mathbf{Q}_{1}=0.1 \mathbf{I}_{60 \times 60}$ and $\mathbf{R}=10$, were used for the LQR synthesis. Fig. 16 shows the time response of the four elastic modal coordinates, and 
Fig. 17 presents the fifth modal coordinate related to the rotation of the trailing-edge control surface. Unstable oscillations grow up to time $t=0.1 \mathrm{~s}$, at which point the controller is switched on until the end of the simulation. Similar to the 2D test case, it is found that the controller designed for the delay free system can suppress the flutter efficiently for the $t_{\mathrm{d}}=0.0$ case. For increasing time delay, the performance of the controller degrades until the capability to suppress the aeroelastic instability is lost for the largest time delay, $t_{\mathrm{d}}=0.0012 \mathrm{~s}$. The values of time delay are smaller in high speed flow than in subsonic flow because of the high frequency oscillations in high speed flow (Huang et al., 2015b). As illustrated next, a new controller designed to account explicitly for the system time delay is needed to ensure a good performance, particularly in the transonic regime.
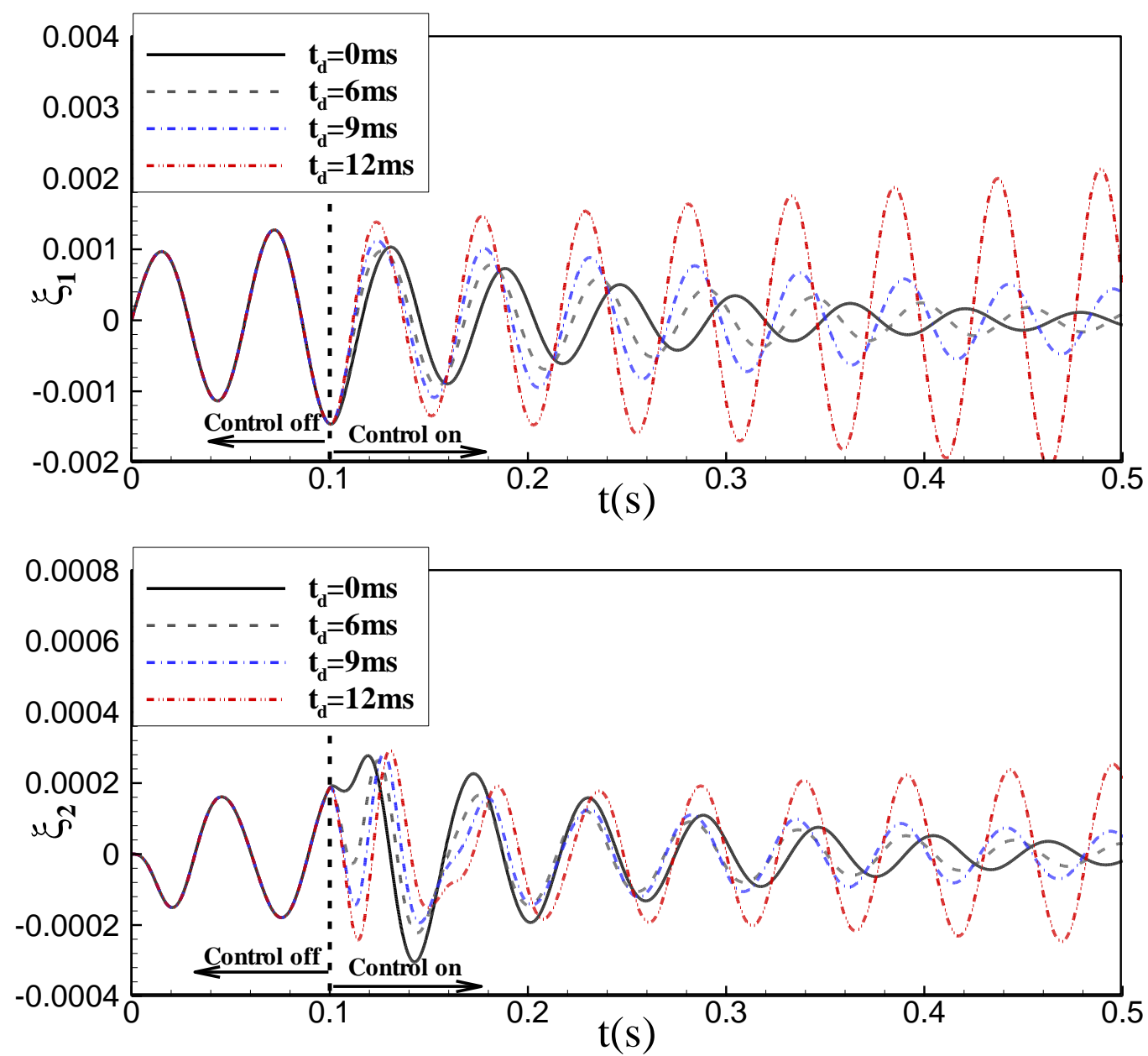

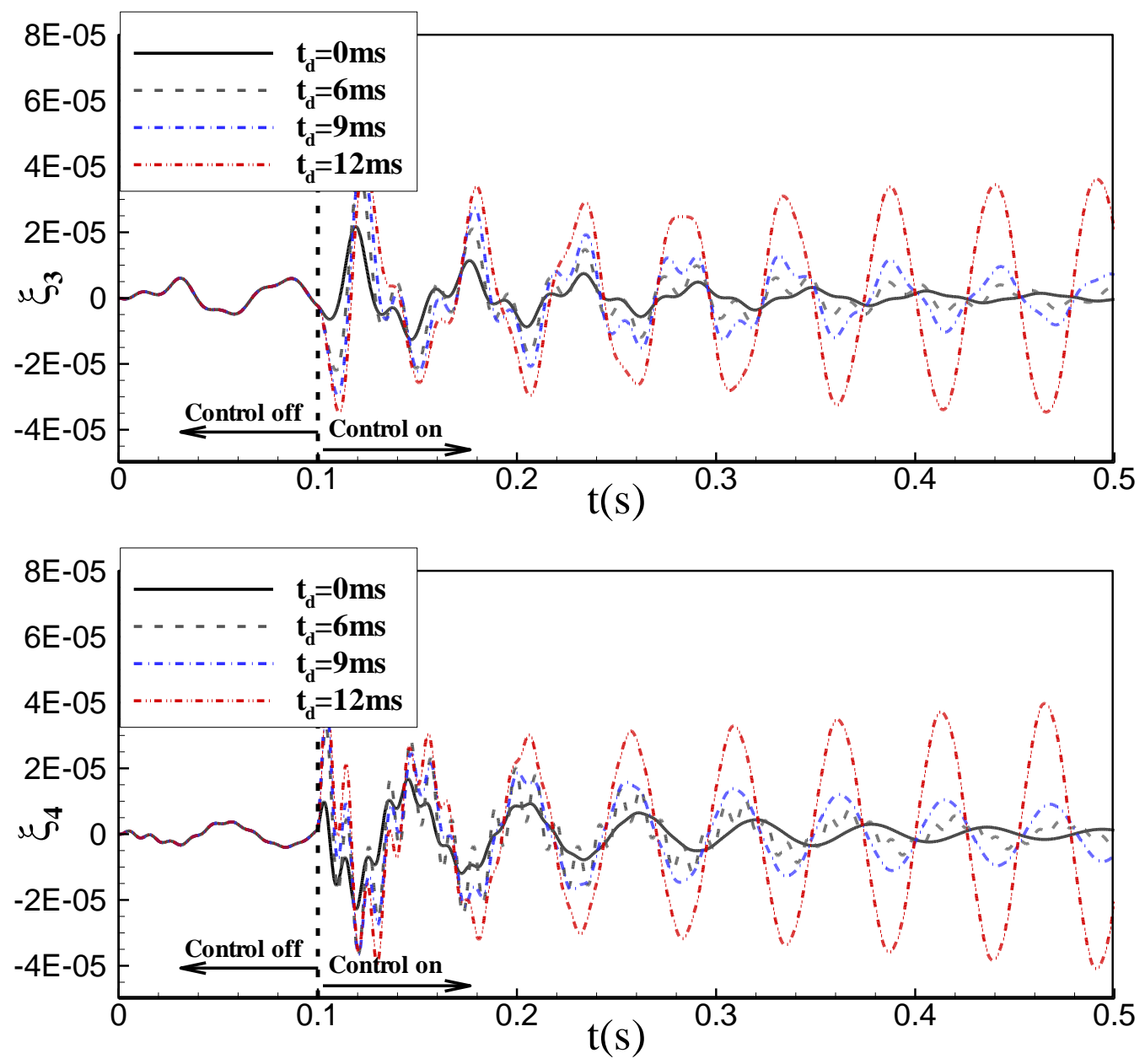

Fig. 16 Closed-loop response in terms of modal coordinates computed by the coupled CFD/CSD method for various control input time delays; feedback controller designed on nominal (delay-free) plant (AGARD 445.6 wing, $\mathrm{M}=0.901, \mathrm{~V}=310.0 \mathrm{~m} / \mathrm{s}$; flutter $\mathrm{VF}=288.4 \mathrm{~m} / \mathrm{s}$ )

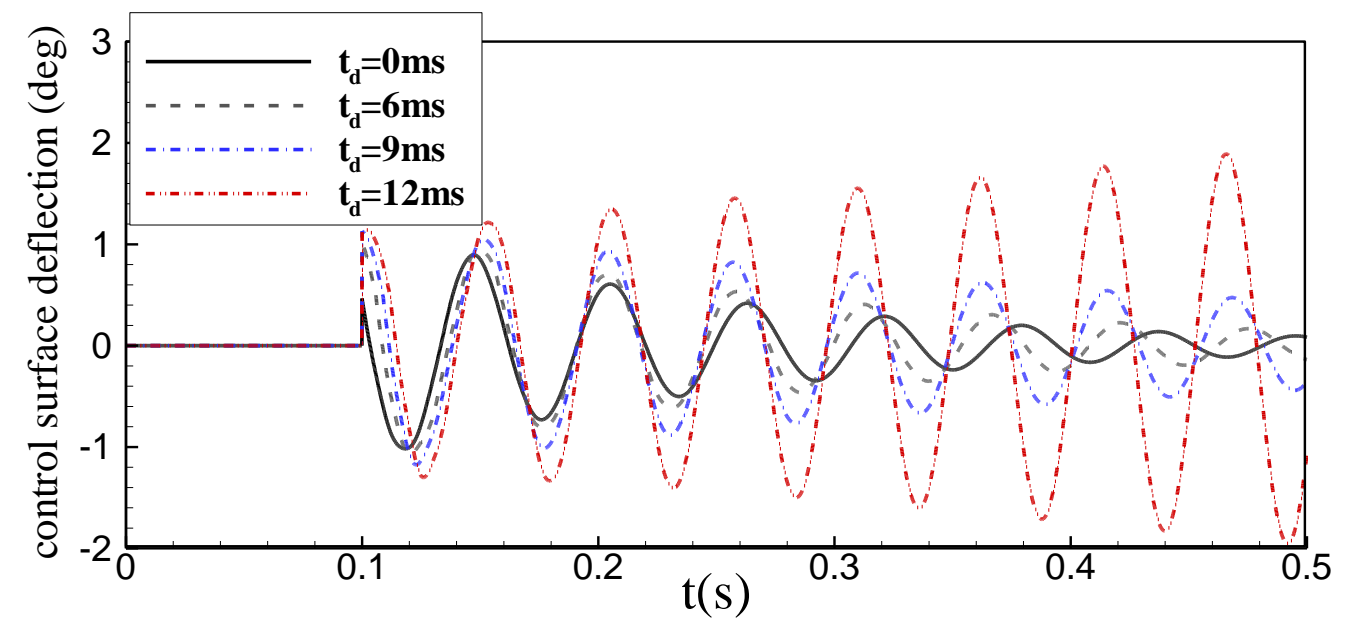

Fig. 17 Trailing-edge control surface rotation computed by the coupled CFD/CSD method for various control input time delays; feedback controller designed on nominal (delay-free) plant (AGARD 445.6 wing, $\mathrm{M}=0.901, \mathrm{~V}=310.0 \mathrm{~m} / \mathrm{s}$; flutter $\mathrm{VF}=288.4 \mathrm{~m} / \mathrm{s}$ ) 


\subsubsection{Active Flutter Suppression with Time-delay}

Fig. 16 and Fig. 17 have shown that a feedback controller, designed on a delayfree system, is inadequate to suppress the aeroelastic instabilities for a time delay larger than $t_{\mathrm{d}}=12 \mathrm{~ms}$. A new time delay controller was then designed for the case of a time delay $t_{\mathrm{d}}=12 \mathrm{~ms}$. The response in terms of modal coordinates is shown in Fig. 18 . The controller was switched on at $t=0.1 \mathrm{~s}$ within the simulation.

Next, the aero-servo-elastic ROM was employed to determine the maximum time delay that causes the controller, which was designed for the case of a time delay $t_{\mathrm{d}}=12 \mathrm{~ms}$, to fail to suppress the aeroelastic instability. The maximum time delay was found to be $t_{\mathrm{d}}=20.8 \mathrm{~ms}$. For verification purposes, the controller was implemented in closed-loop CFD/CSD simulation setting the time delay at $t_{\mathrm{d}}=20.8 \mathrm{~ms}$. Fig. 19 confirms that the closed-loop aeroelastic motion is turned into constant amplitude oscillations, demonstrating the accuracy of the ROM to identify the loss of effectiveness of the controller.
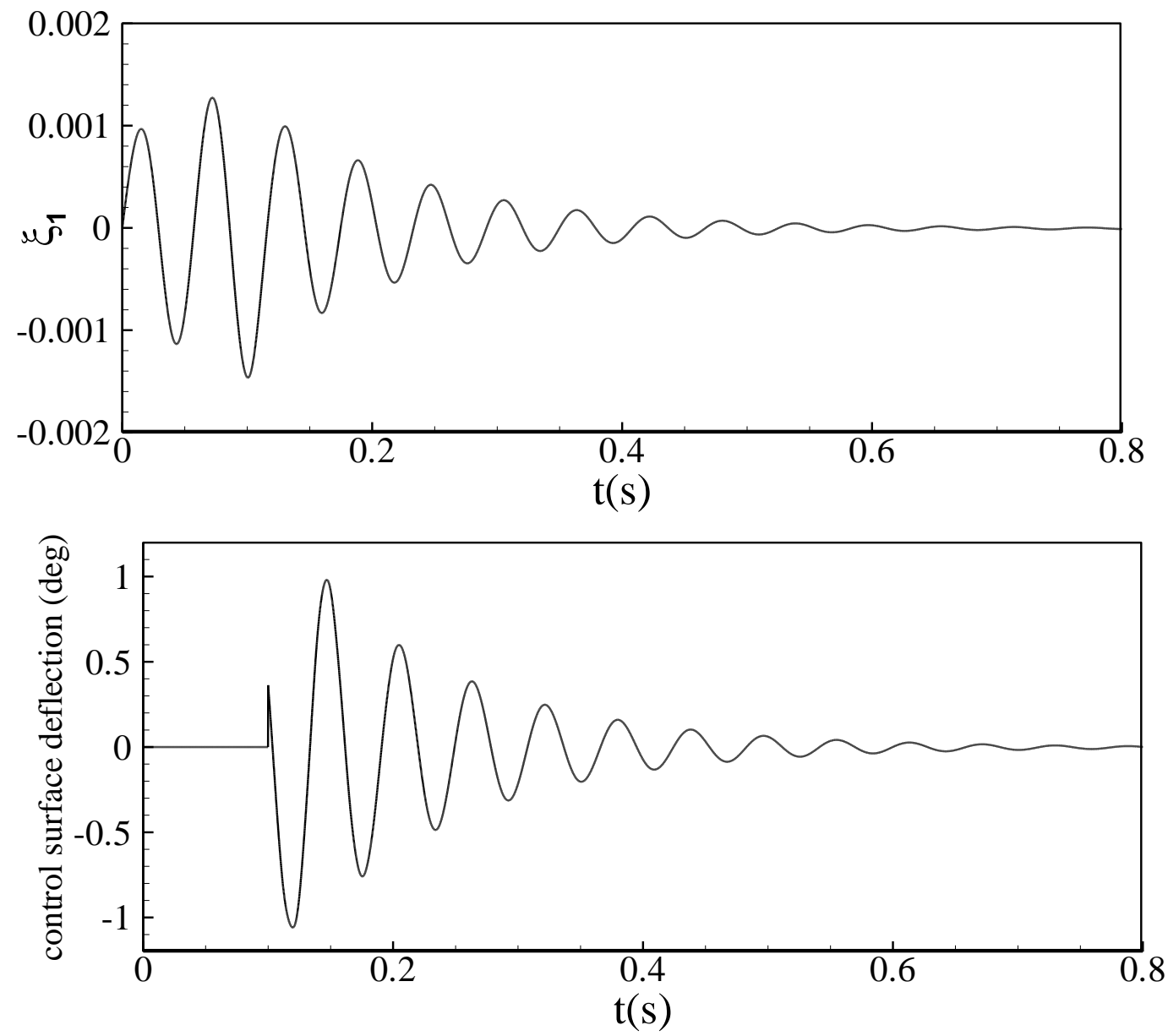

Fig. 18 Closed-loop first modal coordinate and trailing-edge control surface rotation computed by the coupled CFD/CSD method for a control input time delay $t_{\mathrm{d}}=12.0 \mathrm{~ms}$; 
feedback controller designed on time-delayed plant with $t_{\mathrm{d}}=12.0 \mathrm{~ms}$ (AGARD 445.6 wing, $\mathrm{M}=0.901, \mathrm{~V}=310.0 \mathrm{~m} / \mathrm{s} ;$ flutter $\mathrm{VF}=288.4 \mathrm{~m} / \mathrm{s}$ )
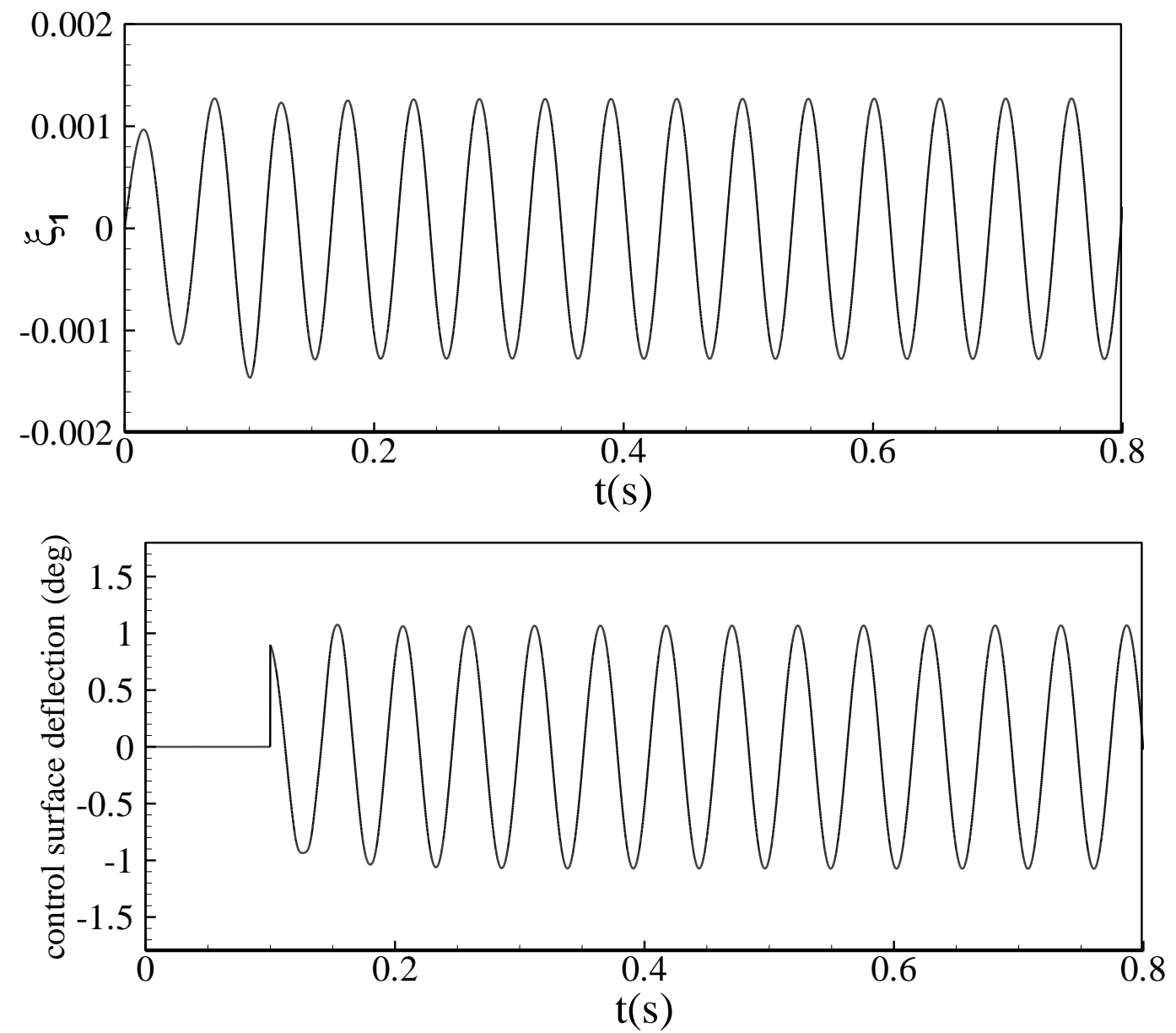

Fig. 19 Closed-loop first modal coordinate and trailing-edge control surface rotation computed by the coupled CFD/CSD method for a control input time delay $t_{\mathrm{d}}=20.8 \mathrm{~ms}$; feedback controller designed on time-delayed plant with $t_{\mathrm{d}}=12.0 \mathrm{~ms}$ (AGARD 445.6 wing, $\mathrm{M}=0.901, \mathrm{~V}=310.0 \mathrm{~m} / \mathrm{s} ;$ flutter $\mathrm{VF}=288.4 \mathrm{~m} / \mathrm{s}$ )

When the time delay increases to $t_{\mathrm{d}}=24 \mathrm{~ms}$, it is not unexpected that the closedloop system becomes unstable, as shown in Fig. 20. A new controller is redesigned using the ROM, and the closed-loop response of the FOM is then presented in Fig. 21. Results indicate that the new controller has a good performance in suppressing wing vibrations when the closed-loop CFD/CSD simulation involves a time delay of $t_{\mathrm{d}}=24 \mathrm{~ms}$.

The various test cases have shown that a controller which is designed to account for time delays can achieve flutter suppression. This raises the question about 
determining with accuracy the time delay of a system, but finding a satisfactory answer to this is out of the scope of this work.
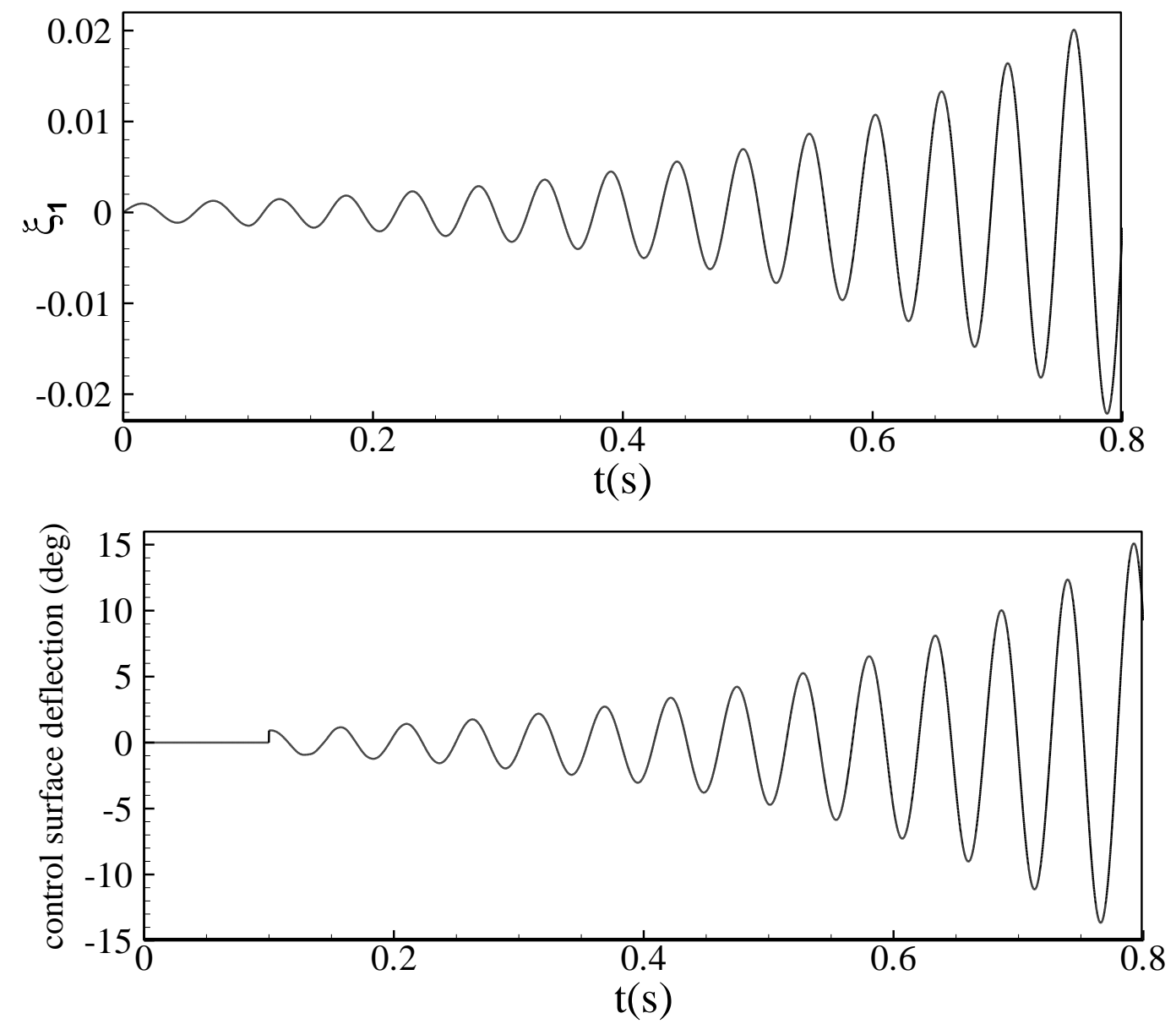

Fig. 20 Closed-loop first modal coordinate and trailing-edge control surface rotation computed by the coupled CFD/CSD method for a control input time delay $t_{\mathrm{d}}=24.0 \mathrm{~ms}$; feedback controller designed on time-delayed plant with $t_{\mathrm{d}}=12.0 \mathrm{~ms}$ (AGARD 445.6 wing, $\mathrm{M}=0.901, \mathrm{~V}=310.0 \mathrm{~m} / \mathrm{s} ;$ flutter $\mathrm{VF}=288.4 \mathrm{~m} / \mathrm{s}$ ) 

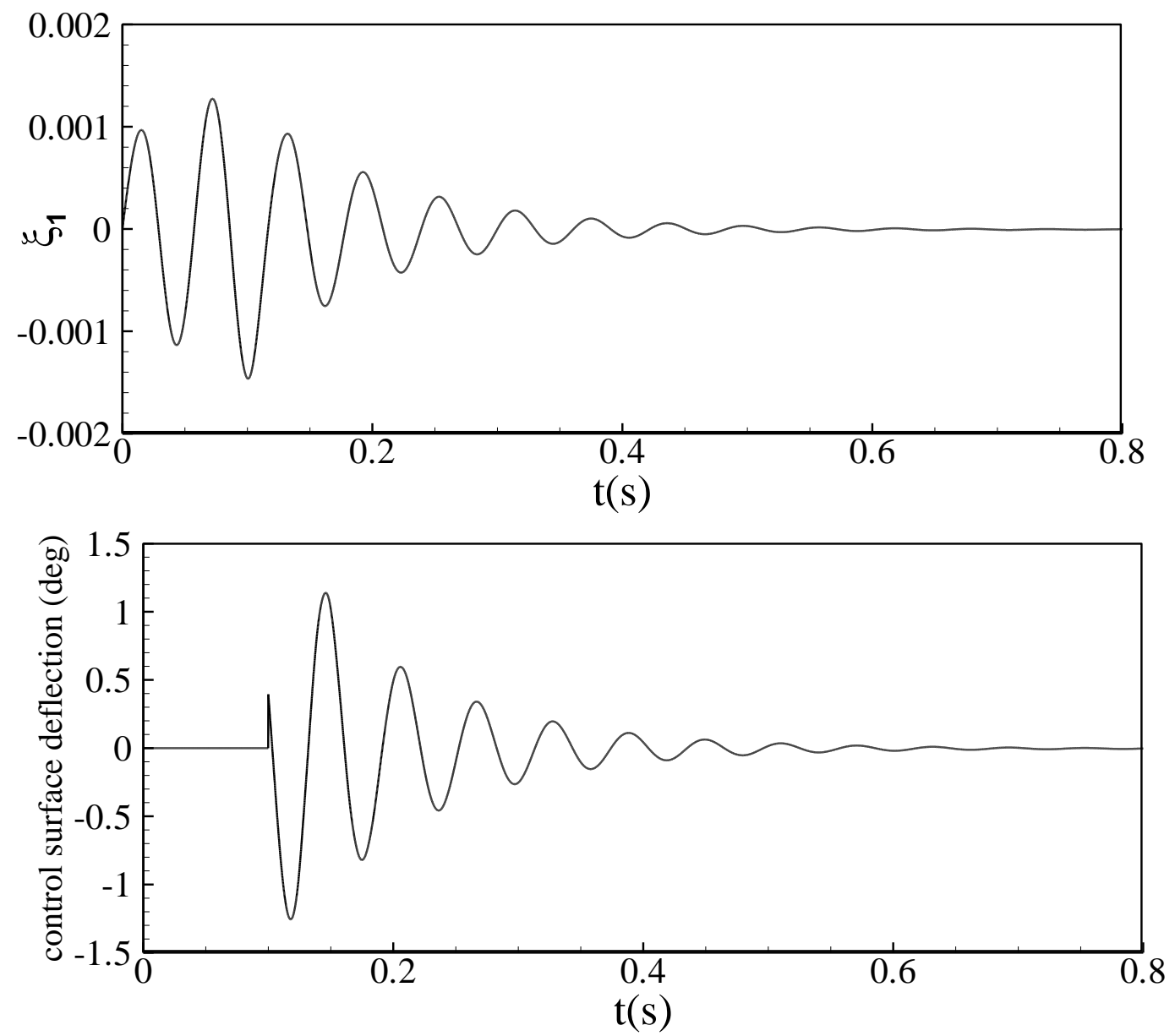

Fig. 21 Closed-loop first modal coordinate and trailing-edge control surface rotation computed by the coupled CFD/CSD method for a control input time delay $t_{\mathrm{d}}=24.0 \mathrm{~ms}$; feedback controller designed on time-delayed plant with $t_{\mathrm{d}}=24.0 \mathrm{~ms}$ (AGARD 445.6 wing, $\mathrm{M}=0.901, \mathrm{~V}=310.0 \mathrm{~m} / \mathrm{s} ;$ flutter $\mathrm{VF}=288.4 \mathrm{~m} / \mathrm{s}$ )

\section{Conclusions}

A unified approach to computational aero-servo-elastic analysis has been demonstrated for a pitch-plunge aerofoil section (NACA0012) and the AGARD 445.6 wing for transonic flows. The advantages of the process, which may be applicable to any generic-purpose coupled computational fluid/structural dynamics framework, are that: a) the generation of a reduced order model of the fluid, which relies on proper orthogonal decomposition combined with balance truncation, is systematic, robust, and efficient; $b$ ) the subsequent generation of a low-order state-space aero-servoelastic reduced order model is straightforward practically, in turn identifying the flutter boundary with excellent accuracy and facilitating the control design synthesis for flutter suppression; and $c$ ) the control strategy, designed on the aero-servo-elastic 
reduced order model, was implemented and tested within the computationally expensive coupled fluid/structural dynamics framework.

Once generated, the state-space aeroelastic reduced order model predicted the stability boundary of all test cases at no extra cost, but with high accuracy in the predictions. The model was used to design a feedback controller, based on a linear quadratic regular strategy within the scope of this work, which was found to provide excellent flutter suppression characteristics. Then, an assessment of the impact of control input time delays, between the measurement of the system state and the control actuation, on the stability of the closed-loop aero-servo-elastic plant was carried out. Qualitatively, it was not unexpected to observe a degradation of the control effectiveness on flutter suppression for increasing control input time delay. Quantitatively, it was found that a small control input time delay may lead the closedloop plant to instability: for the NACA0012 at $\mathrm{M}=0.8$ and speed about $17 \%$ above the flutter speed, the critical time delay was $t_{\mathrm{d}}=0.0092 \mathrm{~s}$; and for the AGARD 445.6 wing at $\mathrm{M}=0.901$ and speed about $8 \%$ above the flutter speed, the critical time delay was $t_{\mathrm{d}}=0.009 \mathrm{~s}$.

As the state-space aeroelastic reduced order model formulation includes naturally the control input time delay, without need for re-generation of the model, feedback controllers were designed with a known time delay in the plant. It was found that the new controllers were able to suppress flutter for larger time delays than the critical value. Finally, it was shown that the aero-servo-elastic reduced order model may be used to identify, for a given feedback controller, the critical value of control input time delay at which the closed-loop aero-servo-elastic system loses its stability. It is worth noting that the question about the identification of the control input time delay of a given control system, which is herein assumed known or a parameter, is not addressed as out of the scope of the current research.

\section{Acknowledgements}

This work was supported by the National Basic Research Program of China (2013CB03570202), the National Natural Science Foundation of China (No. 11272005, 11472206, 11371288 and 11511130053), and the Basic Research foundations for the Central Universities (2014XJJ0126). Andrea Da Ronch acknowledges the financial support by the Royal Academy of Engineering under the Newton Research Collaboration Programme (NRCP1516/1/105). 


\section{Appendix A}

Substituting Eq.(21) into Eq.(20), the discrete system may be written as

$$
\mathbf{x}[(\mathrm{k}+1) \Delta \mathrm{t}]=\mathrm{e}^{\mathbf{A}_{a s e} \Delta t} \mathbf{x}(\mathrm{k} \Delta \mathrm{t})+\int_{0}^{\Delta t} e^{\mathbf{A}_{a s e} \eta} \mathbf{B}_{a s e} \mathbf{u}_{c}[(\mathrm{k}-l+m) \Delta t-\eta] \mathrm{d} \eta+\int_{0}^{\Delta t} e^{\mathbf{A} \eta} d \eta \mathbf{E}_{a s e} w(\mathrm{k} \Delta \mathrm{t})
$$

Rearranging Eq.(31), one obtains

$$
\begin{aligned}
\mathbf{x}[(k+1) \Delta t] & =\mathrm{e}^{\mathbf{A}_{a s e} \Delta t} \mathbf{x}(k \Delta t)+\int_{0}^{m \Delta t} e^{\mathbf{A}_{\text {ass } \eta}} \mathbf{B}_{\text {ase }} \mathbf{u}_{c}[(k-l+m) \Delta t] \mathrm{d} \eta \\
& +\int_{m \Delta t}^{\Delta t} e^{\mathbf{A}_{\text {ase }} \eta} \mathbf{B}_{\text {ase }} \mathbf{u}_{c}[(k-l) \Delta t] \mathrm{d} \eta+\int_{0}^{\Delta t} e^{\mathbf{A}_{\text {ase } \eta}} d \eta \mathbf{E}_{\text {ase }} w(k \Delta t)
\end{aligned}
$$

Assuming the control surface rotation is constant in the integration, Eq.(32) becomes

$$
\mathbf{x}[(k+1) \Delta t]=\mathbf{A}_{d} \mathbf{x}(k \Delta t)+\mathbf{B}_{d 1} \mathbf{u}_{c}[(k-l) \Delta t]+\mathbf{B}_{d 2} \mathbf{u}_{c}[(k-l+1) \Delta t]+\mathbf{E}_{d} w(k \Delta t)
$$

where

$$
\begin{aligned}
& \mathbf{A}_{d}=e^{\mathbf{A}_{a s e} \Delta t} \\
& \mathbf{B}_{d 1}=\int_{m \Delta t}^{\Delta t} e^{\mathbf{A}_{\text {ase }} \eta} d \eta \mathbf{B}_{a s e}=\mathbf{A}_{a s e}^{-1}\left[e^{\mathbf{A}_{a s e} \Delta t}-e^{\mathbf{A}_{a s e} m \Delta t}\right] \mathbf{B}_{a s e}=\mathbf{A}_{a s e}^{-1}\left[\mathbf{A}_{d}-\mathbf{A}_{d}^{m}\right] \mathbf{B}_{a s e} \\
& \mathbf{B}_{d 2}=\int_{0}^{m \Delta t} e^{\mathbf{A}_{\text {ase }} \eta} d \eta \mathbf{B}_{a s e}=\mathbf{A}_{a s e}^{-1}\left[e^{\mathbf{A}_{a s e} m \Delta t}-\mathbf{I}_{n}\right] \mathbf{B}_{a s e}=\mathbf{A}_{a s e}^{-1}\left[\mathbf{A}_{d}^{m}-\mathbf{I}_{n}\right] \mathbf{B}_{a s e} \\
& \mathbf{E}_{d}=\int_{0}^{\Delta t} e^{\mathbf{A}_{\text {ase }} \eta} d \eta \mathbf{E}_{\text {ase }}=\mathbf{A}_{a s e}^{-1}\left[e^{\mathbf{A}_{\text {ase }} \Delta t}-\mathbf{I}_{n}\right] \mathbf{E}_{\text {ase }}=\mathbf{A}_{\text {ase }}^{-1}\left[\mathbf{A}_{d}-\mathbf{I}_{n}\right] \mathbf{E}_{\text {ase }}
\end{aligned}
$$

Finally, Eq.(33) may be written in the form of Eq.(22).

\section{References}

Alder, M., 2015. Development and Validation of a Fluid-Structure Solver for Transonic Panel Flutter. AIAA Journal 53(12), 3509-3521.

Alonso, J., Jameson, A., 1994. Fully-implicit Time-Marching Aeroelastic Solutions, AIAA Paper 19940056, 32th AIAA Aerospace Sciences Meeting and Exhibit, Reno,NV,U.S.A, Jan.

Amsallem, D., 2010. Interpolation on manifolds of CFD-based fluid and finite element-based structural reduced-order models for on-line aeroelastic predictions. Stanford University, State of California Badcock, K.J., Woodgate, M.A., Richards, B.E., 2004. Hopf bifurcation calculations for a symmetric airfoil in transonic flow. AIAA Journal 42(5), 883-892.

Chen, G., Li, Y., Yan, G., 2010. A nonlinear POD reduced order model for limit cycle oscillation prediction. Science China Physics, Mechanics and Astronomy 53(7), 1325-1332.

Chen, G., Sun, J., Li, Y.-M., 2012a. Adaptive reduced-order-model-based control-law design for active flutter suppression. Journal of Aircraft 49(4), 973-980.

Chen, G., Sun, J., Li, Y.M., 2012b. Active flutter suppression control law design method based on balanced proper orthogonal decomposition reduced order model. Nonlinear Dynamics 70(1), 1-12.

Chen, G., Wang, X., Li, Y., 2014. A reduced-order-model-based multiple-in multiple-out gust alleviation control law design method in transonic flow. Science China Technological Sciences 57(2), 368-378.

Chen, Y., Xu, J., 2013. Applications of the integral equation method to delay differential equations. Nonlinear Dynamics 73(4), 2241-2260. 
Da Ronch, A., Badcock, K.J., Wang, Y., Wynn, A., Palacios, R., 2012. Nonlinear model reduction for flexible aircraft control design, AIAA Paper 2012-4404, AIAA Atmospheric Flight Mechanics Conference, Minneapolis, Minnesota, 13-16 Aug, doi:10.2514/6.2012-4404.

Da Ronch, A., McCracken, A., Badcock, K., Widhalm, M., Campobasso, M., 2013a. Linear frequency domain and harmonic balance predictions of dynamic derivatives. Journal of Aircraft 50(3), 694-707.

Da Ronch, A., Tantaroudas, N., Badcock, K., 2013b. Reduction of nonlinear models for control applications, AIAA Paper 2013-1491, 54th AIAA/ASME/ASCE/AHS/ASC Structures, Structural Dynamics, and Materials Conference, Boston, MA, 8-11 Apr, doi:10.2514/6.2013-1491.

Da Ronch, A., Tantaroudas, N., Jiffri, S., Mottershead, J., 2014. A Nonlinear Controller for Flutter Suppression: from Simulation to Wind Tunnel Testing, AIAA Paper 2014-0345, AIAA Science and Technology Forum and Exposition, National Harbor, MD, 13-17 Jan, doi:10.2514/6.2014-0345.

Da Ronch, A., Tantaroudas, N., Timme, S., Badcock, K., 2013c. Model reduction for linear and nonlinear gust loads analysis, AIAA Paper 2013-1492, 54th AIAA/ASME/ASCE/AHS/ASC Structures, Structural Dynamics, and Materials Conference, Boston, MA, 8-11 Apr, doi:10.2514/6.2013-1492.

Dai, H., Yue, X., Xie, D., Atluri, S.N., 2014a. Chaos and chaotic transients in an aeroelastic system. Journal of Sound \& Vibration 333(26), 7267-7285.

Dai, H., Yue, X., Yuan, J., Atluri, S.N., 2014b. A time domain collocation method for studying the aeroelasticity of a two dimensional airfoil with a structural nonlinearity. Journal of Computational Physics 270(3), 214-237.

Dai, H.L., Abdelkefi, A., Wang, L., Liu, W.B., 2014c. Control of cross-flow-induced vibrations of square cylinders using linear and nonlinear delayed feedbacks. Nonlinear Dynamics 78(2), 907-919.

Dai, H.L., Abdelkefi, A., Wang, L., Liu, W.B., 2015. Time-delay feedback controller for amplitude reduction in vortex-induced vibrations. Nonlinear Dynamics 80(1-2), 59-70.

Feng, L., Cai, J., Zhu, Y., Tsai, H., F. Wong, A., 2001. Calculation of wing flutter by a coupled fluidstructure method. Journal of Aircraft 38(2), 334-342.

Fichera, S., Jiffri, S., Wei, X., Da Ronch, A., Tantaroudas, N., Mottershead, J., 2014. Experimental and numerical study of nonlinear dynamic behaviour of an aerofoil, ISMA 3609-3618, ISMA International Conference on Noise and Vibration Engineering, Leuven, Belgium, 15-19 Sep.

Ghoreyshi, M., Cummings, R.M., Ronch, A.D., Badcock, K.J., 2013. Transonic aerodynamic load modeling of X-31 Aircraft Pitching Motions. AIAA Journal 51(10), 2447-2464.

Hall, K.C., Thomas, J.P., Dowell, E.H., 2000. Proper orthogonal decomposition technique for transonic unsteady aerodynamic flows. AIAA Journal 38(10), 1853-1862.

Harder, R.L., Desmarais, R.N., 1972. Interpolation using surface splines. Journal of Aircraft 9(2), 189191.

Hu, H., Dowell, E.H., Virgin, L.N., 1998a. Resonances of a Harmonically Forced Duffing Oscillator with Time Delay State Feedback. Nonlinear Dynamics 15(4), 311-327.

Hu, H., Dowell, E.H., Virgin, L.N., 1998b. Stability estimation of high dimensional vibration systems under state delay feedback control. Journal of Sound and Vibration 214(3), 497-511.

Hu, H., Wang, Z., 2002. Dynamics of Controlled Mechanical Systems with Delayed Feedback. Springer, Berlin

Huang, R., Hu, H., Zhao, Y., 2012. Designing active flutter suppression for high-dimensional aeroelastic systems involving a control delay. Journal of Fluids \& Structures 34(4), 33-50.

Huang, R., Li, H., Hu, H., Zhao, Y., 2015a. Open/Closed-Loop Aeroservoelastic Predictions via Nonlinear, Reduced-Order Aerodynamic Models. AIAA Journal 53(7), 1812-1824.

Huang, R., Qian, W., Hu, H., Zhao, Y., 2015b. Design of active flutter suppression and wind-tunnel tests of a wing model involving a control delay. Journal of Fluids \& Structures 55409-427.

Librescu, L., Marzocca, P., Silva, W.A., 2005. Aeroelasticity of 2-D lifting surfaces with time-delayed feedback control. Journal of Fluids \& Structures 20(2), 197-215.

Lieu, T., Farhat, C., Lesoinne, M., 2005. POD-based aeroelastic analysis of a complete F-16 configuration: ROM adaptation and demonstration, AIAA Paper 2005-2295, 46th AIAA/ASME/ASCE/AHS/ASC Structures, Structural Dynamics and Materials Conference, Austin, Texas, 18 - 21 April.

Liu, B., Hu, H., 2010. Group delay induced instabilities and Hopf bifurcations, of a controlled double pendulum. International Journal of Non-Linear Mechanics 45(4), 442-452.

Lucia, D.J., Beran, P.S., Silva, W.A., 2004. Reduced-order modeling: new approaches for computational physics. Progress in Aerospace Sciences 40(1), 51-117.

Marzocca, P., Librescu, L., Silva, W., 2012. Nonlinear Open-/Closed-Loop Aeroelastic Analysis of Airfoils via Volterra Series. AIAA Journal 42(4), 673-686. 
Olgac, N., Holm-Hansen, B.T., 1994. A Novel Active Vibration Absorption Technique: Delayed Resonator. Journal of Sound \& Vibration 176(1), 93-104.

Papatheou, E., Tantaroudas, N., Da Ronch, A., Cooper, J., Mottershead, J.E., 2013. Active control for flutter suppression: an experimental investigation, IFASD Paper 2013-2018D, International Forum on Aeroelasticity and Structural Dynamics (IFASD), Bristol, U.K, 24-27 Jun.

Tantaroudas, N., Ronch, A.D., Badcock, K., Palacios, R., 2015. Model Order Reduction for Control Design of Flexible Free-Flying Aircraft, AIAA Paper 2015-0240, AIAA Science and Technology Forum and Exposition, Kissimmee, FL, 05-09 Jan, doi:10.2514/6.2015-0240.

Tantaroudas, N., Ronch, A.D., Gai, G., Badcock, K., Palacios, R., 2014. An Adaptive Aeroelastic Control Approach by using Nonlinear Reduced Order Models, AIAA Paper 2014-2590, AIAA Aviation Conference, Atlanta, GA, 16-20 Jun, doi:10.2514/6.2014-2590.

Tantaroudas, N.D., Da Ronch, A., 2015. Nonlinear reduced order aeroservoelastic analysis of very flexible aircraft, in: Marques, P., Da Ronch, A., Tsach, S. (Eds.), Novel Concepts in Unmanned Aircraft Aerodynamics, Flight Stability, and Control. John Wiley \& Sons Inc, Chichester, U.K, ISBN13: 978-1118928684.

Timme, S., Badcock, K., 2009. Oscillatory behavior of transonic aeroelastic instability boundaries. AIAA Journal 47(6), 1590-1592.

Timme, S., Badcock, K., Da Ronch, A., 2013. Linear reduced order modelling for gust response analysis using the DLR-TAU code, IFASD Paper 2013-2036A, International Forum on Aeroelasticity and Structural Dynamics (IFASD), Bristol, U.K, 24-27 Jun.

Tsai, H., F. Wong, A., Cai, J., Zhu, Y., Liu, F., 2001. Unsteady flow calculations with a parallel multiblock moving mesh algorithm. AIAA journal 39(6), 1021-1029.

Van Leer, B., 1979. Towards the ultimate conservative difference scheme. V. A second-order sequel to Godunov's method. Journal of computational Physics 32(1), 101-136.

Wang, Y., Da Ronch, A., Ghandchi-Tehrani, M., Li, E., 2014. Adaptive feedforward control design for gust loads alleviation and LCO suppression, ICAS Paper 2014-0463, 29th Congress of the International Council of the Aeronautical Sciences, St. Petersburg, Russia, 07-12 Sep.

Wang, Y., Li, F., Da Ronch, A., 2015. Adaptive Feedforward Control Design for Gust Loads Alleviation of Highly Flexible Aircraft, AIAA Paper 2015-2243, AIAA Atmospheric Flight Mechanics Conference, Dallas, Texas, 22-26 Jun, doi:10.2514/6.2015-2243.

Xie, D., Xu, M., Dai, H., Dowell, E.H., 2014a. Observation and evolution of chaos for a cantilever plate in supersonic flow. Journal of Fluids \& Structures 50271-291.

Xie, D., Xu, M., Dowell, E.H., 2014b. Proper Orthogonal Decomposition Reduced-Order Model for Nonlinear Aeroelastic Oscillations. AIAA Journal 52(2), 229-241.

Xu, B., Zhang, W., Ma, J., 2014. Stability and Hopf bifurcation of a two-dimensional supersonic airfoil with a time-delayed feedback control surface. Nonlinear Dynamics 77(3), 1-19.

Yates, C.E., Land, N.S., J. T. Foughner, J., 1963. Measured and Calculated Subsonic and Transonic Flutter Characteristics of a 45-degree Swept-Back Wing Planform in Air and in Freon-12 in the Langley Transonic Dynamics Tunnel, Tech. Rep.No.TND-1616, NASA., Hampton, VA.

Yates Jr, E.C., 1988. AGARD standard aeroelastic configurations for dynamic response I-wing 445.6, ADVISORY GROUP FOR AEROSPACE RESEARCH AND DEVELOPMENT NEUILLY-SURSEINE (FRANCE). DTIC Document

Yingtao, Z., Jingjing, L., Gang, C., Yueming, L., Zhenghong, G., 2013. Efficient Multidisciplinary Aerodynamic Optimization Design Based on Discrete Adjoint Method, AIAA Paper 2013-1504, 54th AIAA/ASME/ASCE/AHS/ASC Structures, Structural Dynamics, and Materials Conference, Boston, Massachusetts, April 8-11.

Yuan, Y., Yu, P., Librescu, L., Marzocca, P., 2004. Aeroelasticity of Time-Delayed Feedback Control of Two-Dimensional Supersonic Lifting Surfaces. Journal of Guidance, Control \& dynamics 27(5), 795-803.

Zhao, Y.H., 2009. Stability of a two-dimensional airfoil with time-delayed feedback control. Journal of Fluids \& Structures 25(1), 1-25.

Zhao, Y.H., 2011. Stability of a time-delayed aeroelastic system with a control surface. Aerospace Science \& Technology 15(1), 72-77.

Zhao, Y.Y., Xu, J., 2007. Effects of delayed feedback control on nonlinear vibration absorber system. Journal of Sound \& Vibration 308(1), 212-230.

Zhou, Q., Chen, G., Li, Y., 2014. A Reduced Order Model Based on Block Arnoldi Merhod for aeroelastic system. International Journal of Applied Mechanics 6(06), 1450069. 